\title{
Higher Order Forward Rate Agreements and the Smoothness of the Term Structure
}

Stefan Jaschke

Version 1.1

November 22, 1998 
Institut für Mathematik

Humboldt-Universität zu Berlin

Unter den Linden 6

10099 Berlin, Germany

tel: $\quad+49(30) 20935834$

fax: $\quad+49(30) 20935848$

mail: jaschke@mathematik.hu-berlin.de

http://wws.mathematik.hu-berlin.de/ jaschke/

\section{Credits}

The research on this paper was carried out at the Sonderforschungsbereich 373 at Humboldt University Berlin. Parts of the work were done at Stockholm Business School. I'd like to thank Tomas Björk for his hospitality and discussions. Financial support by the Deutsche Forschungsgemeinschaft and the Deutsche Akademische Austauschdienst is gratefully acknowledged. 


\begin{abstract}
This paper proposes linear higher order conditions on the term structure that allow to compute valuation bounds for any deterministic cash stream. Starting from bounds on the forward rate curve and its derivatives, which are nonlinear in the discount factors, we derive linear conditions that are only slightly less restrictive than the nonlinear conditions. The linearization of the term structure constraints has two advantages. First, the valuation bounds can be computed by highly developed LP solvers. Second, the constraints have an economic meaning as auxiliary cash streams. Thus, price discrepancies can be easily translated into profitable trading strategies.

Depending on the choice of the constraints on the forward rate curve the valuation bounds for cash streams can be very wide or very close. Arbitrage bounds are a special case of our general valuation bounds. On the other end of the extreme, the valuation bounds on the term structure itself behave like quadratic splines in the forward rate curve if the third order parameters are chosen in a restrictive way.

The higher order conditions on the term structure are related to extremal event statistics of short-term interest rates. This puts the resulting valuation bounds conceptually close to risk measures like value at risk. In fact, the proposed method is an example of a coherent risk measure in a sense slightly more general as in the seminal paper by Artzner, Delbaen, Eber, and Heath (1998).

Methods that "calibrate" a single price system to observed prices abound. Needed are valuation bounds that are based solely on economic assumptions. The valuation bounds under the linear higher order conditions on the term structure generalize arbitrage bounds and provide a sharper method when the arbitrage principle is too weak.
\end{abstract}

Keywords: term structure of interest rates, smoothing splines, forward rates, arbitrage, dominance, linear programming

JEL classification: E43, C14, C61

Mathematics Subject Classification: primary: 90-08, 90A12; secondary:

90C05, 62G07, 62-07, 62-09 


\section{Higher Order Forward Rate Agreements and the Smoothness of the Term Structure}

\section{Introduction}

\subsection{Practical Problems}

The term structure of interest rates is the functional relation between time to maturity $\tau$ and the current market price $V(\tau)$ of a guaranteed payment of 1 unit in $\tau$ years time. Instead of the discount function $V(\tau)$, the term structure can equivalently be given as the zero-bond yield curve $r(\tau)=-\log (V(\tau)) / \tau$ or the forward rate curve $f(\tau)=-\frac{d}{d \tau} \log (V(\tau))$. The term structure of interest rates defines a linear price system for the set of deterministic cash streams by virtue of the present value equation. If $z=\left(z_{0}, \ldots, z_{n}\right)$ denotes a cash stream of payments due at the times $\tau_{0}<\ldots<\tau_{n}$, then

$$
\pi(z)=\sum_{j=1}^{n} z_{j} V\left(\tau_{j}\right)
$$

is the present value of $z$ with respect to $V$.

In many practical problems in fixed income markets the term structure of interest rates plays a pivotal role. One of the most important problems is the valuation of cash streams, which may not be traded, relative to the prices of liquidly traded securities, so called benchmark securities. Given the prices of the benchmark securities, one estimates the term structure (by linear regression in the present value equation) and uses that to value all other cash streams.

Another application is cheap/dear-analysis, where one fits a term structure to all or a subset of the traded securities and then interprets the difference between the observed price and the present value under the estimated term structure as an indication whether a security is relatively dear or cheap.

There are many ways to estimate the term structure. Here is an overview:

1. Parametric Methods These include polynomial approximations of the forward rate curve (Chambers et al.; 1984), a piecewise constant forward rate curve with fixed knots (Coleman et al.; 1992), and several special methods with relatively few parameters (Nelson and Siegel (1987) and Svensson (1994)).

\section{Non-parametric Methods}

(a) Regression Splines McCulloch $(1971,1975)$ uses quadratic and cubic regression splines for the discount function. The number of knots is a function of the number of observations. The term structure is estimated by ordinary least squares (linear) regression. 
(b) Orthogonal Polynomials Schaefer (1982) uses Bernstein polynomials to estimate the derivative of the discount function.

(c) Smoothing Splines Tanggaard (1995); Fisher et al. (1995); Bekdache and Baum (1994); Waggoner (1997) use smoothing splines to estimate the forward rate curve. The bandwidth (smoothing parameter) may be selected by cross validation.

Pichler (1995), Anderson et al. (1996), and Schich (1996) give a good overview of the many different methods in use.

Different estimation methods, especially different bandwidth choices in non-parametric estimation, give different answers to the question whether a security is "relatively cheap". In practice, one often uses several methods and believes that a bond is "cheap" only if all methods agree on that. An alternative to the use of different term structure estimators with many hidden statistical assumptions is to employ one method that provides valuation bounds that are based on explicit economic assumptions only.

Depending on the economic assumptions one is willing to make, the valuation bounds are more or less stringent. Independently of their exact economic nature, valuation bounds have a certain mathematical structure in common: they can be defined in terms of a dominance relation for cash streams and term structure packets.

\subsection{Dominance Relations and Term Structure Packets}

Let $0=\tau_{0}<\tau_{1}<\ldots<\tau_{n}$ be a sequence of payment dates, which are not necessarily equidistant. Cash streams are vectors $z=\left(z_{0}, \ldots, z_{n}\right)$ that pay the amount $z_{j}$ at time $\tau_{j}$. A dominance relation $\succeq$ is a partial ordering on the set of cash streams that is compatible with the vector space structure and such that the set $\{z \mid z \succeq 0\}$ is a nonempty closed convex polyhedral cone. ${ }^{1}$ Read " $y \succ z$ " as " $y$ is strictly preferred to $z$ ", or " $y$ is better than $z$, independently of personal preferences".

A price system on the set of all cash streams on $\left(\tau_{0}, \ldots, \tau_{n}\right)$ is now given by a vector $u=\left(V\left(\tau_{0}\right), \ldots, V\left(\tau_{n}\right)\right)$. As we keep the set of payment dates fixed, we call such a discretized discount function also a term structure. A term structure $u$ is admissible if $u^{\prime} z=\sum_{j=0}^{n} u_{j} z_{j} \geq 0$ for all $z \succeq 0$. Note that the set of admissible term structures is a closed convex polyhedral cone,

\footnotetext{
${ }^{1} \mathrm{~A}$ partial ordering on a set $E$ is a subset $M$ of the cross product $E \times E$ such that (i) $x \in E$ implies $(x, x) \in M$ and (ii) $(x, y) \in M$ and $(y, z) \in M$ imply $(x, z) \in M$. Instead of $(x, y) \in M$ one usually writes $x \succeq y$. $x \succ y$ is defined as ' $x \succeq y$ and not $y \succeq x$ '. A partial ordering on a vector space is compatible with the vector space structure if (i) $x \succeq y$ if and only if $x-y \succeq 0$ and (ii) $x \succeq 0$ implies $\alpha x \succeq 0$ for all $\alpha \geq 0$. As a matter of fact, any closed convex cone $K \subset E$ defines a vector space-compatible partial ordering by $x \succeq y \stackrel{\text { def }}{=} x-y \in K$. Although the theory works with closed convex cones in general, we restrict ourselves to polyhedral cones here since this is the only kind we use for the practical implementation.
} 
which we denote by $K$. Obviously, $\{z \mid z \preceq 0\}$ and $K$ are polar cones $^{2}$. So, a dominance relation for cash streams can also be defined by starting with a closed convex polyhedral cone $K$ of admissible term structures and setting $x \succeq y \stackrel{\text { def }}{=} \quad u^{\prime}(x-y) \geq 0 \quad \forall u \in K$.

Consider now a set of fixed income securities indexed by $i$ that pay the amount $P_{i j}$ at time $\tau_{j}$. Given buying prices $S_{i}$, each security defines a cash stream $\left(-S_{i}, P_{i 1}, \ldots, P_{i n}\right)$. ( $S_{i}$ may include transaction costs.) If short-selling is possible, the security also defines the cash stream $\left(s_{i},-P_{i 1}, \ldots,-P_{i n}\right)$, where $s_{i}$ is the net receipt from short-selling the security. These cash streams define a cash flow matrix $C=\left(\begin{array}{c|c}-S & P \\ \hline s & -P\end{array}\right)$.

A transaction is a vector $x \geq 0$, with the meaning that $x_{i}$ units of the cash stream $i$ are bought. $C^{\prime} x$ is the cash stream that is generated by the transaction $x$. We call $x$ a good deal of the first type if $C^{\prime} x \succ 0$. We call $x$ a good deal of the second type $e^{3}$ if $C^{\prime} x \succeq 0$ and $\left(C^{\prime} x\right)_{0}>0$ (i.e., free lunch at time 0 and no future obligations.) We say that $N G D$ holds, if there is no good deal of the second type.

An important example of a dominance relation is the arbitrage relation that is defined by $K_{a}=\left\{u \mid u_{0} \geq u_{1} \geq \ldots \geq u_{n} \geq 0\right\}$, and which is implicit in the works of Schaefer $(1981,1982)$ and the arbitrage theory of Dermody and Rockafellar (1991, 1995). NGD corresponds to their WNA (weak no arbitrage). Another important example is the dominance relation that is defined by $K=$ $\left\{u \mid \underline{f}_{j} \leq u_{j} / u_{j+1}-1 \leq \bar{f}_{j} \forall j, u \geq 0\right\}$, which was used by Hodges and Schaefer (1977) and Ronn (1987). ( $\underline{f}_{j}$ and $\bar{f}_{j}$ are bounds on the implied forward rate for the interval $\left[\tau_{j}, \tau_{j+1}\right]$.)

The traded cash streams $C$ and the dominance relation $\succeq$ imply good deal bounds for the time-0 price of any cash stream $z$ :

$$
\begin{aligned}
& \bar{\pi}(z)=\min _{p, x}\left\{p \mid(p, 0, \ldots, 0)+C^{\prime} x \succeq z, x \geq 0, p \in \mathbf{R}\right\}, \\
& \underline{\pi}(z)=\max _{p, x}\left\{p \mid(p, 0, \ldots, 0)-C^{\prime} x \preceq z, x \geq 0, p \in \mathbf{R}\right\} .
\end{aligned}
$$

If somebody offers to buy the cash stream $z$ for a price $\pi(z)>\bar{\pi}(z)$, we can form a good deal by selling $z$ and hedging that obligation with a transaction $x$ that realizes the minimum in (2). We earn the immediate gain $\pi(z)-\bar{\pi}(z)$ and own a cash stream $(\bar{\pi}(z), 0, \ldots, 0)+C^{\prime} x-z$ that is at least as good as the zero cash stream in the sense of $\succeq$. Analogously, we can form a good deal, if somebody offers to sell the cash stream $z$ for a lower price than $\underline{\pi}(z)$.

Define the set of term structures that are consistent with the traded cash streams $C$ and the dominance relation defined by $K$ as the set

$$
D=\left\{u \mid C u \leq 0, u \in K, u_{0}=1, u \geq 0\right\} .
$$

\footnotetext{
${ }^{2}$ The polar cone $K^{\perp}$ of a cone $K$ is defined as $K^{\perp}=\left\{y \mid y^{\prime} z \leq 0 \forall z \in K\right\}$. For any closed convex cone $K\left(K^{\perp}\right)^{\perp}=K$ holds. See for example (Bertsekas; 1995, p.580)

${ }^{3}$ The wording is patterned after (Ingersoll; 1987, p.53).
} 
Note that the condition $C u \leq 0$ is the same as $s \leq P u \leq S$ (with $u_{0}=1$ ). In words, a term structure is consistent if for each security the "theoretical" price (under $u$ ) lies between the prices for short-selling and buying.

\section{Theorem 1 Fundamental Theorem}

1.) NGD holds if and only if $D$ is nonempty.

2.) In this case, the good deal bounds can also be computed as the maximal and minimal present values of $z$ with respect to the term structure packet $D$ :

$$
\bar{\pi}(z)=\max _{u \in D} u^{\prime} z \quad \text { and } \quad \underline{\pi}(z)=\min _{u \in D} u^{\prime} z .
$$

Proof. This is just the duality theorem of linear programming. One merely has to check that $\bar{\pi}(z)=\max _{u \in D} u^{\prime} z$ is in fact the dual of the problem (2) (see (4) and (5) in the next section).

\subsection{Applications of the Arbitrage Principle}

Hodges and Schaefer (1977), Schaefer (1982), and Rasch (1996) use the arbitrage principle to prove that "there is something wrong with the present value equation". More precisely, heterogeneous taxation and short-selling constraints lead to the effect that certain bonds are held only by certain investors (their clientele), and there is no term structure (price system) that values all cash streams correctly - not even approximately. In other words, the arbitrage principle can be used to prove that there is a clientele effect in both quantities and prices (Dybvig and Ross; 1986). Standard regression approaches cannot be used for this, since they are invariably based on statistical assumptions and there is no economic reasoning to decide when residuals are "too large". 4

A second application is portfolio optimization under consideration of taxes as done by Hodges and Schaefer (1977). If there are tax clientele effects, it may not be obvious that certain portfolios are suboptimal. The arbitrage principle can also be used to identify bonds that a certain investor should definitely not hold ("dominated bonds").

A third application is to estimate tax-specific term structures, as in (Schaefer; 1981).

There are, however, several problems and short-comings of the arbitrage principle in applications. The arbitrage bounds for a cash stream $z$ may be too wide to be useful as a valuation tool. This is the case when the cash streams that can be generated $\left(\left\{C^{\prime} x \mid x \geq 0\right\}\right)$ do not compare well with the cash stream $z$ in terms of $\succeq$. As a consequence, the arbitrage principle may fail to uncover relative mispricing. As a special case, it may fail to identify tax clientele effects for bonds that have a very low coupon (Rasch; 1996).

It is an old idea (Hodges and Schaefer; 1977; Ronn; 1987) to sharpen the arbitrage relation by introducing bounds on implied forward rates. The mission

\footnotetext{
${ }^{4}$ There is, however, a relation between minimax regression and arbitrage theory, as described in (Jaschke; 1998).
} 
of this paper is to propose higher order conditions that are approximately the same as placing bounds on the first and the second derivative of the forward rate curve. It turns out that these conditions are very effective in the sense that the resulting valuation bounds are much closer than arbitrage bounds. Still, these bounds are non-parametric, free of any statistical assumptions and based on explicit economic assumptions only. Although the computational cost of these bounds is much higher than for traditional term structure methods, the fact that they boil down to linear programming problems and the recent advances in LP solver technology (CPLEX, PCx, SoPlex) allow to compute these valuation bounds in a few seconds.

Section 2 motivates higher order forward rate agreements and explains how they are related to bounds on the derivatives of the forward rate curve. Section 3 derives the exact higher order conditions based on the conditions on short-term rate movements as opposed to the approximate relation that was used in section 2 to motivate higher order FRAs. Section 4 addresses some numerical issues. Section 5 presents descriptive statistics of German short-term interest rates. These are needed to determine sensible bounds for the forward rate curve and its derivatives. Section 6 explores applications of higher order forward rate conditions to various valuation problems.

\section{Higher Order Forward Rate Agreements}

The primal and dual optimization problems for the upper good deal bound can be written as

$$
\begin{aligned}
\bar{\pi}(z) & =\min _{p, x, h}\left\{p \mid(p, 0, \ldots, 0)+C^{\prime} x+A^{\prime} h \geq z, x \geq 0, h \geq 0, p \text { free }\right\} \\
& =\max _{u}\left\{u^{\prime} z \mid\left(\begin{array}{l}
C \\
A
\end{array}\right) u \leq 0, u \geq 0, u_{0}=1\right\}
\end{aligned}
$$

for any polyhedral cone $K$ of the form $K=\{u \mid A u \leq 0, u \geq 0\}$. The cone of admissible term structures

$$
K^{(1)}=\left\{u \mid \underline{f}_{j} \leq u_{i} / u_{i+1}-1 \leq \bar{f}_{j}, u \geq 0\right\}
$$

that was implicitly used by Hodges and Schaefer (1977) can be written as

$$
K^{(1)}=\left\{u \mid A^{(1)} u \leq 0, u \geq 0\right\}
$$

with

$$
A^{(1)}=\left(\begin{array}{rrrr}
\ddots & & & 0 \\
& 1 & -\left(1+\bar{f}_{j}\right) & \\
& -1 & 1+\underline{f}_{j} & \\
0 & & & \ddots
\end{array}\right) .
$$

This can now be interpreted in three ways. 
1. $\underline{f}_{j}$ and $\bar{f}_{j}$ are bounds on the interest $R_{j}$ for the future interval $\left[\tau_{j}, \tau_{j+1}\right]$.

If the primal variable $h_{k}$ that corresponds to the row $\left(\ldots,-1,1+\underline{f}_{j}, \ldots\right)$ in $A^{(1)}$ is positive in an optimal solution of (4), this means that then one should invest $h_{k}$ units in a money market account at time $\tau_{j}$. One gets back $\left(1+R_{j}\right) h_{k}$ and can consume $h_{k}\left(R_{j}-\underline{f}_{j}\right)$ since only $h_{k}(1+$ $\left.\underline{f}_{j}\right)$ is needed to cover $z_{j}$. Analogously, the row $\left(\ldots, 1,-\left(1+\bar{f}_{j}\right), \ldots\right)$ corresponds to the borrowing of money from $\tau_{j}$ to $\tau_{j+1}$. In other words, $h$ is interpreted as a roll-over strategy in a money market account.

If one wants to believe that $\underline{f}_{j} \leq R_{j} \leq \bar{f}_{j}$ holds with probability one, the resulting good deals could still be called arbitrage transactions.

2. The rows in $A$ can be interpreted as auxiliary cash streams.

The matrix $\left(\begin{array}{l}C \\ A\end{array}\right)$ can be interpreted as a new cash flow matrix where $C$ contains the traded cash streams that we can readily get in a liquid market. The matrix $A$ contains auxiliary cash streams that are not traded, but that we are confident we can somehow get in the OTC market. In the case of $A^{(1)}$, the cash streams are forward rate agreements (FRAs). It might be, for example, that there are no liquidly traded FRAs for a certain market segment, say, US dollars from year 2027 to year 2028, but we are confident we can find a counter-party for an FRA if we offer to borrow at $15 \%$ p.a. or lend at $0.5 \%$ p.a.

This interpretation allows sharper bounds than the first interpretation. We may be successful in closing an over-the-counter deal borrowing $15 \%$ from 2027 to 2028 , but we may not believe that the money market rate in that future time interval will be below $15 \%$ with probability one.

3. The condition $A^{(1)} u \leq 0$ places bounds on the first derivative of the (discrete) discount function. This is approximately the same as the condition that the forward rate curve $\tau_{j} \mapsto f\left(\tau_{j}\right)$ is bounded by $\underline{f}_{j}$ and $\bar{f}_{j}$.

Assume for a moment equidistant payment dates $\left(\tau_{j}=j\right)$ and define the "discrete forward rate curve"

$$
f(j)=\log u_{j}-\log u_{j+1}
$$

Since $e^{x} \approx 1+x$ for $x$ near 0 , the original condition

$$
1+\underline{f}_{j} \leq e^{f(j)} \leq 1+\bar{f}_{j}
$$

is approximately the same as

$$
\underline{f}_{j} \leq f(j) \leq \bar{f}_{j}
$$


Since forward rates are related to the first derivative of the discount function, we call $A^{(1)} u \leq 0$ the first order conditions of the term structure.

With the third interpretation in mind, the second and third order conditions $A^{(k)} u \leq 0$ for equidistant payment dates are easily written as

$$
\begin{aligned}
& A^{(2)}=\left(\begin{array}{rrrrr}
\ddots & & & & 0 \\
& -1 & 2 & -\left(1+\bar{f}_{j}^{(2)}\right) & \\
& 1 & -2 & 1+\underline{f}_{j}^{(2)} & \\
0 & & & & \ddots
\end{array}\right) \quad \text { and } \\
& A^{(3)}=\left(\begin{array}{rrrrrr}
\ddots & & & & & 0 \\
& 1 & -3 & 3 & -\left(1+\bar{f}_{j}^{(3)}\right) & \\
& -1 & +3 & -3 & 1+\underline{f}_{j}^{(3)} & \\
0 & & & & & \ddots
\end{array}\right) . \\
& A^{(2)} u \leq 0 \text { is equivalent to } \\
& 1+\underline{f}_{j}^{(2)} \leq 2 e^{f(j+1)}-e^{f(j+1)+f(j)} \leq 1+\bar{f}_{j}^{(2)}, \forall j,
\end{aligned}
$$

which is approximately the same as

$$
\underline{f}_{j}^{(2)} \leq f(j+1)-f(j) \leq \bar{f}_{j}^{(2)}, \forall j .
$$

$A^{(3)} u \leq 0$ is equivalent to

$$
1+\underline{f}_{j}^{(3)} \leq 3 e^{f(j+2)}-3 e^{f(j+2)+f(j+1)}+e^{f(j+2)+f(j+1)+f(j)} \leq 1+\bar{f}_{j}^{(3)}, \forall j,
$$

which is approximately the same as

$$
\underline{f}_{j}^{(3)} \leq f(j+2)-2 f(j+1)+f(j) \leq \bar{f}_{j}^{(3)}, \forall j .
$$

In this sense, the second and third order conditions $A^{(k)} u \leq 0, k=2,3$ are approximately the same as placing bounds on the first and second derivative of the forward rate curve.

In view of the second interpretation, the rows of $A^{(2)}$ and $A^{(3)}$ can be interpreted as higher order forward rate agreements. The cash streams can be constructed by going long and short in FRAs of consecutive periods. If higher order FRAs would be traded directly, the higher order forward rate $f_{j}^{(3)}$ that makes the present value of the cash stream $\left(\ldots, 1,-3,3,-\left(1+f_{j}^{(3)}\right), \ldots\right)$ zero, would measure the third derivative of the discount function $V(\tau)$ at $\tau=j$.

In terms of the first interpretation, one can show in a similar fashion as above that the second order condition is approximately the same as

$$
\underline{f}_{j}^{(2)} \leq R_{j+1}-R_{j} \leq \bar{f}_{j}^{(2)}, \forall j,
$$


i.e., changes in future short-term rates are bounded. The third order condition is approximately the same as

$$
\underline{f}_{j}^{(3)} \leq R_{j+2}-2 R_{j+1}+R_{j} \leq \bar{f}_{j}^{(3)}, \forall j,
$$

i.e., changes in future short-term rate changes are bounded.

In order to determine appropriate bounds $\left(\underline{f}_{j}^{(k)}, \bar{f}_{j}^{(k)}\right)_{k=1,2,3}$, one can proceed in two ways. One possibility is to fix a grid size, say 3 months, and compute empirical distributions of the accumulated values of the cash streams $(1,-1),(1,-2,1)$, and $(1,-3,3,-1)$ with respect to historic 3 -month interest rates. The empirical distributions can then be used to directly specify the bounds $\left(\underline{f}_{j}^{(k)}, \bar{f}_{j}^{(k)}\right)_{k=1,2,3}$. Preliminary tests with equidistant grids showed that with moderate grid sizes ( $\geq 30$ days), the resulting term structure packets may contain highly non-smooth curves. (They are "smooth on the grid", but nonsmooth in between.) For smaller grid sizes ( $\leq 15$ days), the computational cost becomes significant. When the bounds $\left(\underline{f}_{j}^{(k)}, \bar{f}_{j}^{(k)}\right)_{k=1,2,3}$ are specified directly, one also has to ensure consistency $\left(\left\{u \mid u_{0}=1, u \geq 0, A^{(k)} u \leq 0, k=1,2,3\right\} \neq\right.$ $\emptyset)$, whose preconditions are not obvious.

The second approach is to start with bounds on the first and second derivative of the forward rate curve and then derive exact ${ }^{5}$ bounds for the higher order forward rates on general grids.

\section{Exact Bounds for Higher Order Forward Rates}

\subsection{Conditions on Future Interest Rates}

Fix a sequence of payment dates $0=\tau_{0}<\tau_{1}<\ldots<\tau_{n}$. (Think of $\tau_{j}$ as time to maturity in years.) Define $\Delta_{j}=\tau_{j+1}-\tau_{j}$. Let $r_{j}$ denote the normalized forward rate for the interval $\left[\tau_{j}, \tau_{j+1}\right]: r_{j}=\frac{1}{\Delta_{j}} \int_{\mathcal{\tau}_{j}}^{\tau_{j+1}} f(s) d s$. In terms of the first interpretation, $r_{j}$ can also be thought of as the continuously compounded rate for the interval $\left[\tau_{j}, \tau_{j+1}\right]$, i.e., 1 unit invested at time $\tau_{j}$ becomes $e^{\Delta_{j} r_{j}}$ at time $\tau_{j+1}$.

The conditions

$$
\begin{array}{rlrl}
a_{j}^{(1)} & \leq & r_{j} & \leq b_{j}^{(1)}, \\
-a_{j}^{(2)} & \leq & \frac{r_{j+1}-r_{j}}{\left(\Delta_{j+1}+\Delta_{j}\right) / 2} & \leq b_{j}^{(2)}, \quad \text { and } \\
-a_{j}^{(3)} & \leq g\left(r_{j+1}, r_{j+2}, r_{j+3}\right) & \leq b_{j}^{(3)}
\end{array}
$$

with

$$
g\left(r_{j+1}, r_{j+2}, r_{j+3}\right)=\frac{\frac{r_{j+2}-r_{j+1}}{\left(\Delta_{j+2}+\Delta_{j+1}\right) / 2}-\frac{r_{j+1}-r_{j}}{\left(\Delta_{j+1}+\Delta_{j}\right) / 2}}{\left(\Delta_{j+2}+\Delta_{j+1}+\Delta_{j}\right) / 3}
$$

\footnotetext{
${ }^{5}$ The precision of the approximation $e^{x} \approx 1+x$ is not sufficient for applications. It was only used to motivate higher order forward rate agreements.
} 
are discretized versions of

$$
\begin{aligned}
a_{s}^{(1)} & \leq f(s) \leq b_{s}^{(1)}, \\
-a_{s}^{(2)} & \leq f^{\prime}(s) \leq b_{s}^{(2)}, \quad \text { and } \\
-a_{s}^{(3)} & \leq f^{\prime \prime}(s) \leq b_{s}^{(3)} .
\end{aligned}
$$

We call (9), (10), and (11) the nonlinear higher order conditions since they are nonlinear in the discount factors $u_{j} \cdot\left(\Delta_{j} r_{j}=\log \left(u_{j}\right)-\log \left(u_{j+1}\right)\right)$. The first order conditions (9) are easily transformed to linear conditions $A^{(1)} u \leq 0$ with

$$
A^{(1)}=\left(\begin{array}{rrrr}
\ddots & & & 0 \\
& 1 & -e^{\Delta_{j} b_{j}^{(1)}} & \\
& -1 & e^{\Delta_{j} a_{j}^{(1)}} & \\
0 & & & \ddots
\end{array}\right) .
$$

The nonlinear second and third order conditions cannot be transformed to linear conditions. The rest of this section deals with the derivation of linear conditions $A^{(2)} u \leq 0$ and $A^{(3)} u \leq 0$ such that the term structure packets defined by the nonlinear conditions are contained in the packets defined by the linear conditions, but the difference is minimal.

\subsection{Second Order Auxiliary Cash Streams}

Given a cash stream $\left(\ldots, 1, z_{j+2}, 0, \ldots\right)$, we are looking for the extremal values of its time- $\tau_{j+3}$ accumulated value

$$
v\left(z_{j+2} ; r_{j+1}, r_{j+2}\right):=\left(e^{\Delta_{j+1} r_{j+1}}+z_{j+2}\right) e^{\Delta_{j+2} r_{j+2}}
$$

subject to the first and second order conditions (9), (10). The resulting second order auxiliary cash streams are then

$$
\begin{aligned}
& \left(\ldots, 1, z_{j+2},-\max _{r_{j+1}, r_{j+2}} v\left(z_{j+2} ; r_{j+1}, r_{j+2}\right), \ldots\right) \text { and } \\
& \left(\ldots,-1,-z_{j+2}, \min _{r_{j+1}, r_{j+2}} v\left(z_{j+2} ; r_{j+1}, r_{j+2}\right), \ldots\right) .
\end{aligned}
$$

The rest of this subsection deals with the computation of the extremal values of (15). For the ease of notation, we set $j=0, \Delta_{12}=\left(\Delta_{1}+\Delta_{2}\right) / 2$, and assume that $a_{j}^{(k)}$ and $b_{j}^{(k)}$ are independent of $j$.

Proposition 2 Under the conditions

$$
\begin{aligned}
a^{(k)}>0, b^{(k)}>0, \forall k, & \\
z_{2} & <-e^{\Delta_{1} b^{(1)}}, \quad \text { and } \\
a^{(2)} \Delta_{12} & <b^{(1)}-a^{(1)}
\end{aligned}
$$


there are only two candidates for $\max _{r_{1}, r_{2}} v\left(z_{2} ; r_{1}, r_{2}\right)$ :

(a) $r_{2}-r_{1}=-a^{(2)} \Delta_{12}, r_{1}=b^{(1)}$,

(b) $\quad r_{2}-r_{1}=-a^{(2)} \Delta_{12}, r_{2}=a^{(1)}$.

Under the conditions (16), (17), and

$$
b^{(2)} \Delta_{12}<b^{(1)}-a^{(1)}
$$

there are only three candidates for $\min _{r_{1}, r_{2}} v\left(z_{2} ; r_{1}, r_{2}\right)$ :

(c) $\quad r_{2}-r_{1}=b^{(2)} \Delta_{12}, r_{1}=a^{(1)}$,

(d) $\quad r_{2}-r_{1}=b^{(2)} \Delta_{12}, e^{\Delta_{1} r_{1}}=-\frac{\Delta_{2} z_{2}}{2 \Delta_{12}}$,

(e) $\quad r_{2}-r_{1}=b^{(2)} \Delta_{12}, r_{2}=b^{(1)}$.

If the point defined by (d) is feasible it is optimal.

All proofs are in the last section.

We have now some freedom to choose $z_{2}$. For the auxiliary cash stream $\left(1, z_{2},-\max v\right)$, we propose to choose $z_{2}=z^{a b}$, which is defined by $v\left(z^{a b} ; r_{1}^{a}, r_{2}^{a}\right)=v\left(z^{a b} ; r_{1}^{b}, r_{2}^{b}\right)$. ( $r_{i}^{a}$ and $r_{i}^{b}$ are defined by (19) and (20), respectively.) As a matter of fact,

$$
z^{a b}=\frac{e^{\Delta_{12}\left(2 a^{(1)}+a^{(2)} \Delta_{1}\right)}-e^{\Delta_{12}\left(2 b^{(1)}-a^{(2)} \Delta_{2}\right)}}{e^{\Delta_{2}\left(b^{(1)}-a^{(2)} \Delta_{12}\right)}-e^{\Delta_{2} a^{(1)}}} .
$$

For the cash stream $\left(-1,-z_{2}, \min v\right)$, we propose to choose $z_{2}$ such that the minimum is attained in case $(\mathrm{d})$, for example

$$
\begin{aligned}
r_{1}^{*} & =0.5\left(a^{(1)}+b^{(1)}-b^{(2)} \Delta_{12}\right) \vee a^{(1)} \wedge\left(b^{(1)}-b^{(2)} \Delta_{12}\right) \\
z_{2}^{*} & =-2 \frac{\Delta_{12}}{\Delta_{2}} e^{\Delta_{1} r_{1}^{*}}
\end{aligned}
$$

\subsection{Third Order Auxiliary Cash Streams}

Given a cash stream $\left(\ldots, 1, z_{j+2}, z_{j+3}, 0, \ldots\right)$, we are looking for the extremal values of its time- $\tau_{j+4}$ accumulated value $v\left(z_{j+2}, z_{j+3} ; r_{j+1}, r_{j+2}, r_{j+3}\right)$

$$
=\left(\left(e^{\Delta_{j+1} r_{j+1}}+z_{j+2}\right) e^{\Delta_{j+2} r_{j+2}}+z_{j+3}\right) e^{\Delta_{j+3} r_{j+3}}
$$

subject to the first, second, and third order conditions (9), (10), (11). The resulting second order auxiliary cash streams are then

$$
\left(\ldots, 1, z_{j+2}, z_{j+3}, \quad-\max _{r_{j+1}, r_{j+2}, r_{j+3}} v\left(z_{j+2}, z_{j+3} ; r_{j+1}, r_{j+2}, r_{j+3}\right), \ldots\right)
$$

and

$$
\left.\left(\ldots,-1,-z_{j+2},-z_{j+3}, \min _{r_{j+1}, r_{j+2}, r_{j+3}} v\left(z_{j+2}, z_{j+3} ; r_{j+1}, r_{j+2}, r_{j+3}\right)\right), \ldots\right) .
$$


The rest of this subsection deals with the computation of the extremal values of (25). For the ease of notation, we set $j=0$ and $\Delta_{12}=\left(\Delta_{1}+\right.$ $\left.\Delta_{2}\right) / 2, \Delta_{23}=\left(\Delta_{2}+\Delta_{3}\right) / 2, \Delta_{123}=\left(\Delta_{1}+\Delta_{2}+\Delta_{3}\right) / 3$.

Proposition 3 Under the conditions (16), (17) (from the previous proposition) and

$$
\begin{aligned}
z_{3} & >-e^{\Delta_{2} b^{(1)}}\left(z_{2}+e^{\Delta_{1} a^{(1)}}\right) \\
\Delta_{23} b^{(2)} & <b^{(1)}-a^{(1)} \\
\Delta_{12} a^{(2)} & <b^{(1)}-a^{(1)} \\
\Delta_{23} a^{(2)} & <b^{(1)}-a^{(1)} \\
\Delta_{12} b^{(2)} & <b^{(1)}-a^{(1)} \\
\Delta_{123} b^{(3)} & <a^{(2)}+b^{(2)} \\
\Delta_{123} a^{(3)} & <a^{(2)}+b^{(2)} \\
\Delta_{23} \Delta_{123} b^{(3)} & <a^{(2)}\left(\Delta_{12}+\Delta_{23}\right) \\
\Delta_{12} \Delta_{123} b^{(3)} & <b^{(2)}\left(\Delta_{12}+\Delta_{23}\right) \\
\Delta_{23} \Delta_{123} a^{(3)} & <b^{(2)}\left(\Delta_{12}+\Delta_{23}\right), \text { and } \\
\Delta_{12} \Delta_{123} a^{(3)} & <a^{(2)}\left(\Delta_{12}+\Delta_{23}\right)
\end{aligned}
$$

the maximum of (25) subject to (9), (10), and (11) is attained on the face defined by $\left\{g\left(r_{1}, r_{2}, r_{3}\right)=b^{(3)}\right\}$. The minimum is attained on the face defined by $\left\{g\left(r_{1}, r_{2}, r_{3}\right)=-a^{(3)}\right\}$.

Proposition 4 Under the conditions of the previous proposition and

$$
z_{3} e^{-\Delta_{2} a^{(1)}} \Delta_{3}^{2}\left(A^{2}+\Delta_{2}^{2} z_{2} e^{-\Delta_{1} b^{(1)}}\right)+z_{2}\left(A \Delta_{3}-B \Delta_{2}\right)^{2}<0
$$

with

$$
A=\Delta_{12}\left(\frac{\Delta_{1}}{\Delta_{23}}+2\right)
$$

and

$$
B=\Delta_{3}-\Delta_{1} \Delta_{12} / \Delta_{23}
$$

$v$ is strictly concave on the planes $\left\{\left(r_{1}, r_{2}, r_{3}\right) \mid g\left(r_{1}, r_{2}, r_{3}\right)=\right.$ const. $\}$. The unique maximum of $v$ is determined by

$$
\begin{aligned}
e^{\Delta_{1} r_{1}} & =z_{2} / x \\
e^{\Delta_{1} r_{1}+\Delta_{2} r_{2}} & =z_{3} / y \\
g\left(r_{1}, r_{2}, r_{3}\right) & =b^{(3)}
\end{aligned}
$$

with

$$
\begin{aligned}
& x=-A / \Delta_{2} \quad \text { and } \\
& y=-B / \Delta_{3}-x,
\end{aligned}
$$


provided that this defines a feasible solution. The minimum is attained at one of the 5 points that are determined by

(a) $r_{2}=b^{(1)}, r_{3}-r_{2}=-\Delta_{23} a^{(2)}$,

(b) $r_{3}=a^{(1)}, r_{3}-r_{2}=-\Delta_{23} a^{(2)}$,

(c) $r_{2}=r_{3}=a^{(1)}$,

(d) $\quad r_{1}=a^{(1)}, r_{2}-r_{1}=-\Delta_{12} b^{(2)}, \quad$ or

(e) $\quad r_{2}=b^{(1)}, r_{2}-r_{1}=-\Delta_{12} b^{(2)}$

and $g\left(r_{1}, r_{2}, r_{3}\right)=-a^{(3)}$.

We have now some freedom to choose $\left(z_{2}, z_{3}\right)$. For the maximum, we propose to take some "intermediate" rates $\left(r_{1}^{*}, r_{2}^{*}, r_{3}^{*}\right)$ like

$$
\begin{aligned}
r_{2}^{*} & =\left(a^{(1)}+b^{(1)}\right) / 2 \wedge\left(b^{(1)}-0.5 \Delta_{123} b^{(3)}\left(\Delta_{12} \vee \Delta_{23}\right)\right) \\
r_{3}^{*} & =r_{2}+\Delta_{23} \Delta_{123} b^{(3)} / 2 \\
r_{1}^{*} & =r_{2}+\Delta_{12} \Delta_{123} b^{(3)} / 2
\end{aligned}
$$

and compute $\left(z_{2}, z_{3}\right)$ by $(38)$ and $(39)$.

For the minimum, we solve the optimization problem

$$
\max _{z_{2}, z_{3}}\left\{-v\left(z_{2}, z_{3} ; r_{1}^{*}, r_{2}^{*}, r_{3}^{*}\right)+\min _{r_{1}, r_{2}, r_{3}} v\left(z_{2}, z_{3} ; r_{1}, r_{2}, r_{3}\right)\right\}
$$

Since $\min _{r_{1}, r_{2}, r_{3}} v\left(z_{2}, z_{3} ; r_{1}, r_{2}, r_{3}\right)$ is piecewise linear in $\left(z_{2}, z_{3}\right)$ and the conditions (17) and (26) are linear in $\left(z_{2}, z_{3}\right)$, the problem (48) can be written as a linear optimization problem (of dimension $6 \times 3$ ) and easily solved at run time. The hyperbolic condition (37) is automatically fulfilled in most cases.

\section{Numerical Issues}

The previous section showed how to compute the auxiliary cash flow matrices

$$
\begin{aligned}
& A^{(2)}=\left(\begin{array}{rrrrr}
\ddots & & & & 0 \\
& -1 & -z_{j+2}^{i} & \min v\left(z_{j+2}^{i} ; \ldots\right) & \\
& 1 & z_{j+2}^{a} & -\max v\left(z_{j+2}^{a} ; \ldots\right) & \\
0 & & & & \ddots
\end{array}\right) \quad \text { and } \\
& A^{(3)}=\left(\begin{array}{rrrrrr}
\ddots & & & & & 0 \\
& -1 & -z_{j+2}^{i} & -z_{j+3}^{i} & \min v\left(z_{j+2}^{i}, z_{j+3}^{i} ; \ldots\right) & \\
& 1 & z_{j+2}^{a} & z_{j+3}^{a} & -\max v\left(z_{j+2}^{a}, z_{j+3}^{a} ; \ldots\right) & \\
0 & & & & & \ddots
\end{array}\right) \text {, }
\end{aligned}
$$


given the grid of payment dates $0=\tau_{0}<\tau_{1}<\ldots<\tau_{n}$ and the parameters $\left(a_{s}^{(k)}, b_{s}^{(k)}\right){ }^{6}$

The valuation problems for an arbitrary cash stream $z$

$$
\max _{u \in D} u^{\prime} z \quad \text { and } \quad \min _{u \in D} u^{\prime} z
$$

with

$$
D=\left\{u \mid C u \leq 0, A^{(k)} u \leq 0(k=1,2,3), u_{0}=1, u \geq 0\right\}
$$

are, unfortunately, notoriously ill-conditioned if the grid of payment dates contains very small distances $\Delta_{j}=\tau_{j+1}-\tau_{j}$ like $\Delta_{j}=1$ day. In such cases both SoPlex and PCx have difficulties solving the valuation problems (49).

In order to get a feeling of how ill-conditioned the problems are we compared the maximum of $v\left(z_{j+2}^{a}, z_{j+3}^{a} ; \ldots\right)$ under the nonlinear first, second, and third order conditions to the maximum of $v\left(z_{j+2}^{a}, z_{j+3}^{a} ; \ldots\right)$ under the linear first and second order conditions $A^{(k)} u \leq 0(k=1,2)$. In other words, how much is gained my adding a specific third order auxiliary cash stream? The difference of the two maxima, divided by the "size" of the cash stream $\left(1+\left|z_{j+2}\right|+\left|z_{j+3}\right|\right)$, can be called the relative improvement of the corresponding third order cash stream.

On a series of practical problems, the relative improvement of third order cash streams was between $10^{-8}$ and $10^{-2}$. By dropping the third order cash streams with a relative improvement below $10^{-6}$ we could verify that the numerical problems stem in fact from those cash streams with a small relative improvement. (But dropping these conditions is not a feasible solution to the numerical problem.) From the sampled data it could also be seen that the relative improvement of a third order cash stream on the dates $\left(\tau_{j}, \tau_{j+1}, \tau_{j+2}, \tau_{j+3}\right)$ is roughly proportionate to

$$
\frac{\Delta_{j} \Delta_{j+1} \Delta_{j+2}}{\Delta_{j}+\Delta_{j+1}+\Delta_{j+2}}
$$

thus small distances in the grid of payment dates are the reason for the numerical problems.

A solution that has been working very well in practice is to compute the third order auxiliary cash streams on quadruples $\left(\tau_{j_{1}}, \tau_{j_{2}}, \tau_{j_{3}}, \tau_{j_{4}}\right)$ of payment dates with $\tau_{j_{i+1}}-\tau_{j_{i}} \geq 30$ days instead of neighboring payment dates $\left(\tau_{j}, \tau_{j+1}, \tau_{j+2}, \tau_{j+3}\right)$. This increases the relative improvement of third order cash streams to about $10^{-5}$, which suffices to avoid numerical instabilities.

The relative improvement of second order cash streams over first order conditions is between $10^{-4}$ and $10^{-1}$, so no special treatment is needed for the second order conditions.

\footnotetext{
${ }^{6}$ See $(15)$ and $(25)$ for the definition of $v(\ldots)$. In words, $v(\ldots)$ is the time- $\tau_{j+3}$ accumulated value of the cash stream $\left(\ldots, 1, z_{j+2}, 0, \ldots\right)$ for the second order and the time- $\tau_{j+4}$ accumulated value of the cash stream $\left(\ldots, 1, z_{j+2}, z_{j+3}, 0, \ldots\right)$ for the third order, respectively.
} 


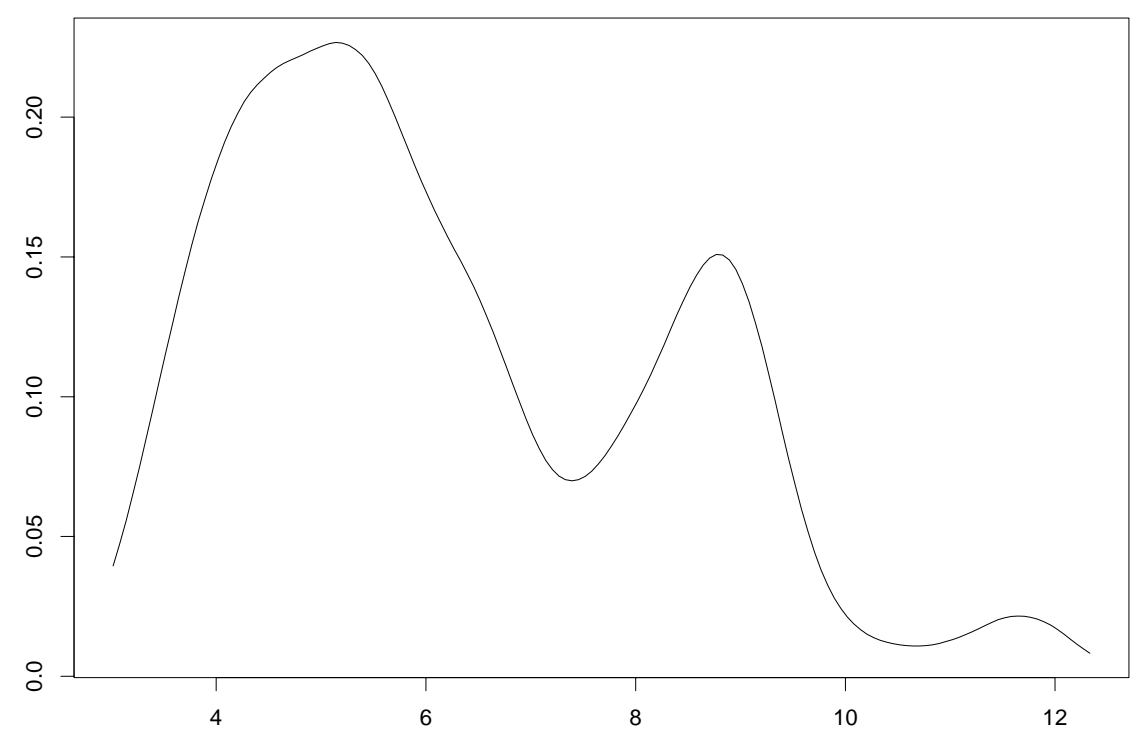

Figure 1: Empirical Distribution of 12-month DM-LIBOR Rates The LIBOR rates are transformed to continuously compounded rates. The density is estimated using the S-Plus function ksmooth with kernel="parzen" and bandwidth=1.

\section{Descriptive Statistics of German Short-Term In- terest Rates}

In this section, we use daily 12-month DM-LIBOR rates from 1975 to $1996^{7}$ to establish some empirical facts about German short-term interest rates. The dataset contains 5551 trading dates. Corresponding to our use of continuously compounded rates, we transform the LIBOR rates as

$$
r_{t}=\log \left(1+12 \text { month-LIBOR }_{t}\right) .
$$

The empirical distribution of short-term interest rates (figure 1) shows two pronounced modes at $5 \%$ and $8.5 \%$. The rates above $10 \%$ come from a rather short period in 1981 .

The following table shows the minimum, the maximum, and several quantiles of the interest rates, yearly changes in the interest rates, and changes in yearly interest rate changes:

\footnotetext{
${ }^{7}$ Thanks to Wolfgang Schmidt, Deutsche Morgan Grenfell.
} 


\begin{tabular}{|l|rrr|rrr|} 
& $\min$ & $1 \%$ & $5 \%$ & $95 \%$ & $99 \%$ & $\max$ \\
\hline$r_{t}$ & 3.02 & 3.21 & 3.62 & 9.43 & 11.78 & 12.33 \\
$r_{t+1}-r_{t}$ & -4.58 & -3.96 & -3.06 & 3.26 & 4.20 & 5.16 \\
$r_{t+2}-2 r_{t+1}+r_{t}$ & -8.43 & -7.18 & -3.76 & 3.62 & 4.52 & 5.64
\end{tabular}

Extremal second order changes are a bit asymmetric. It happened (around 1981), that interest rates went up $4 \%$ in one year and came down $4 \%$ in the following year. An equally sharp dip down and bounce back never happened.

The statistics give a rough feeling of how quickly interest rates can change. They provide rule-of-thumb values for the parameters $\left(a^{(k)}, b^{(k)}\right)$ if one thinks in terms of the first interpretation.

\section{Applications}

In this section we explore two areas of application of good deal bounds where standard arbitrage bounds are too weak to be practically useful:

1. good deal bounds for zero-bonds and forward rate agreements relative to the prices of benchmark securities and

2. identification of tax-clientele effects, when Schaefer's method fails to detect them.

\subsection{Good Deal Bounds for the Term Structure}

One way to assess the effectiveness of valuation bounds is to compute bounds for the prices of a series of zero-bonds (with increasing time to maturity), translate the prices to yields, and then display the resulting bounds for the zero-bond yield curve. Another way is to compute valuation bounds for a series of 6-month forward rate agreements and then display bounds for the curve of 6-month forward rates.

Jaschke (1998) showed that the arbitrage bounds for the zero-bond yield curve are quite close up to 10 years time to maturity, provided that the arbitrage bounds are computed with respect to all outstanding German government bonds (over 100). The problem with this approach is that many of those bonds are not liquidly traded, so "fresh", indicative quotes are put into one basket with non-indicative quotes. When the term structure is estimated for the purpose of market making, practitioners base their estimates solely on the most recent quotes of the most actively traded securities - so-called benchmark securities - because only those quotes contain the latest interest-rate-relevant information.

Bloomberg and Reuters, for example, publish prices of US treasury benchmark bonds on the pages http://www.bloomberg.com/markets/C13.html and http://quotes.reuters.com/qUSBMK.html, respectively. On May 12, 1998 , both pages contained the same set of bonds, namely the current 6 -month 


\begin{tabular}{|l|r|c|} 
security id & $\begin{array}{c}\text { time to maturity } \\
\text { in years }\end{array}$ & $\begin{array}{c}\text { daily trading } \\
\text { volume }\end{array}$ \\
\hline 113505 & 9.7 & 89 \\
113506 & 30.0 & 85 \\
114125 & 4.5 & 71 \\
113681 & 1.9 & 50 \\
114118 & 2.8 & 48 \\
113503 & 9.2 & 36
\end{tabular}

Table 1: Most Actively Traded Government Bonds

The trading volume is the average daily trading volume on the German stock exchanges in million DM face value in the period April 6 - April 30, 1998.

and 1-year T-bills, the current 2-, 3-, 5-, and 10-year T-notes, and the current 30-year bond, except that Bloomberg also included the 3-month T-bill. It turns out that the same maturities are most actively traded in the German government bond market. Table 1 shows the average daily trading volume of the 6 most actively traded German government bonds in the period April 6 - April 30, $1998 .^{8} 113505,113506,114125$, and 113681 are the current (onthe-run) securities with $10,30,5$, and 2 years time to maturity. 114118 is a bobl (5-year bond) that obviously served as a kind of a 3-year benchmark. 113503 is the old ${ }^{9} 10$-year bond and leads a long list of bonds that are traded in quantities of about 30 million DM per day.

In the following, we explore the good deal bounds for zero-bonds and FRAs relative to the five most actively traded bonds from table 1 . Prices are from the Frankfurt stock exchange of April 28, 1998.

Figure 2 shows the arbitrage bounds ${ }^{10}$ for the zero-bond yield curve. Unlike the arbitrage bounds that are computed w.r.t. the prices of all outstanding government bonds, the arbitrage bounds relative to the benchmark bonds are not close enough to be anyhow useful.

In the next step, we impose an upper bound on the implied forward rates as in Hodges and Schaefer (1977), setting $b^{(1)}=0.2$. Figure 3 shows the resulting bounds for the zero-bond yield curve as well as the bounds for the 6 -month forward rate curve.

Adding the second order conditions with $a^{(2)}=b^{(2)}=0.04$ improves the bounds for the term structure significantly, especially the bounds for the forward rate curve (figure 4). Adding the third order conditions with $a^{(3)}=b^{(3)}=$ 0.04 again improves the bounds (figure 5 ).

Up to now, the conditions $\left(a^{(k)}, b^{(k)}\right)$ that we used were rather conservative,

\footnotetext{
${ }^{8}$ We have no data on BuBills as they are are not traded at the stock exchange.

${ }^{9}$ The bond that was issued just before the current on-the-run bond of a certain maturity is called the "old" bond.

${ }^{10}$ I.e., $a^{(1)}=0$ and $b^{(1)}=a^{(k)}=b^{(k)}=\infty, k=2,3$.
} 


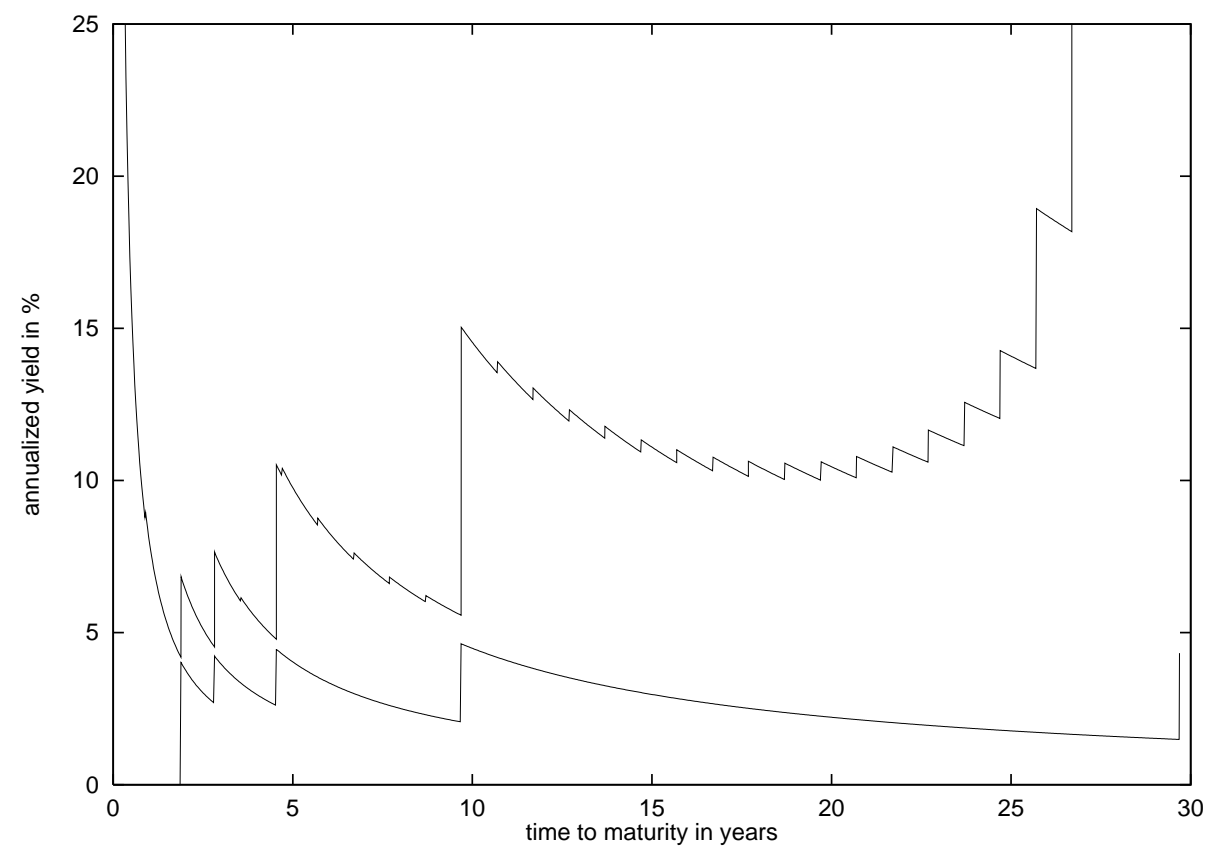

Figure 2: Arbitrage Bounds for the Zero-Bond Yield Curve

so the resulting bounds (figure 5) can still be interpreted as being supported by "almost arbitrage" strategies that may loose only in some rare cases of extreme interest rate movements - in the sense of the first interpretation and with the statistical results of section 5. Looking at figure 5 and taking the viewpoint of the second interpretation we might convince ourselves that we would be able to enter into higher order forward rate agreements according to

$$
\begin{aligned}
& \left(a^{(1)}, b^{(1)}\right)=(2.5 \%, 8 \%) \\
& \left(a^{(2)}, b^{(2)}\right)=(2 \%, 2 \%) \\
& \left(a^{(3)}, b^{(3)}\right)=(1 \%, 1 \%) .
\end{aligned}
$$

The resulting bounds are shown in figure 6 .

Now, we are going to pull all stops and ask (in the spirit of the third interpretation) what the closest reasonable bounds for the forward rate curve and its derivatives are. One can see from figure 6 that the forward rate curve is increasing at about $0.5 \%$ per year at the short end. So we are on the save side when we set $b^{(2)}=1 \%$. But the forward rate cannot increase at that rate over 30 years, so we make the upper bound on the first derivative dependent on time to maturity:

$$
b_{s}^{(2)}=1 \% \cdot 2^{-s / 10},
$$

where $s$ is measured in years. (This means that $f^{\prime}$ is smaller than $0.125 \%$ at the long end.) Since it looks as if the forward rate curve is upward sloping, we 

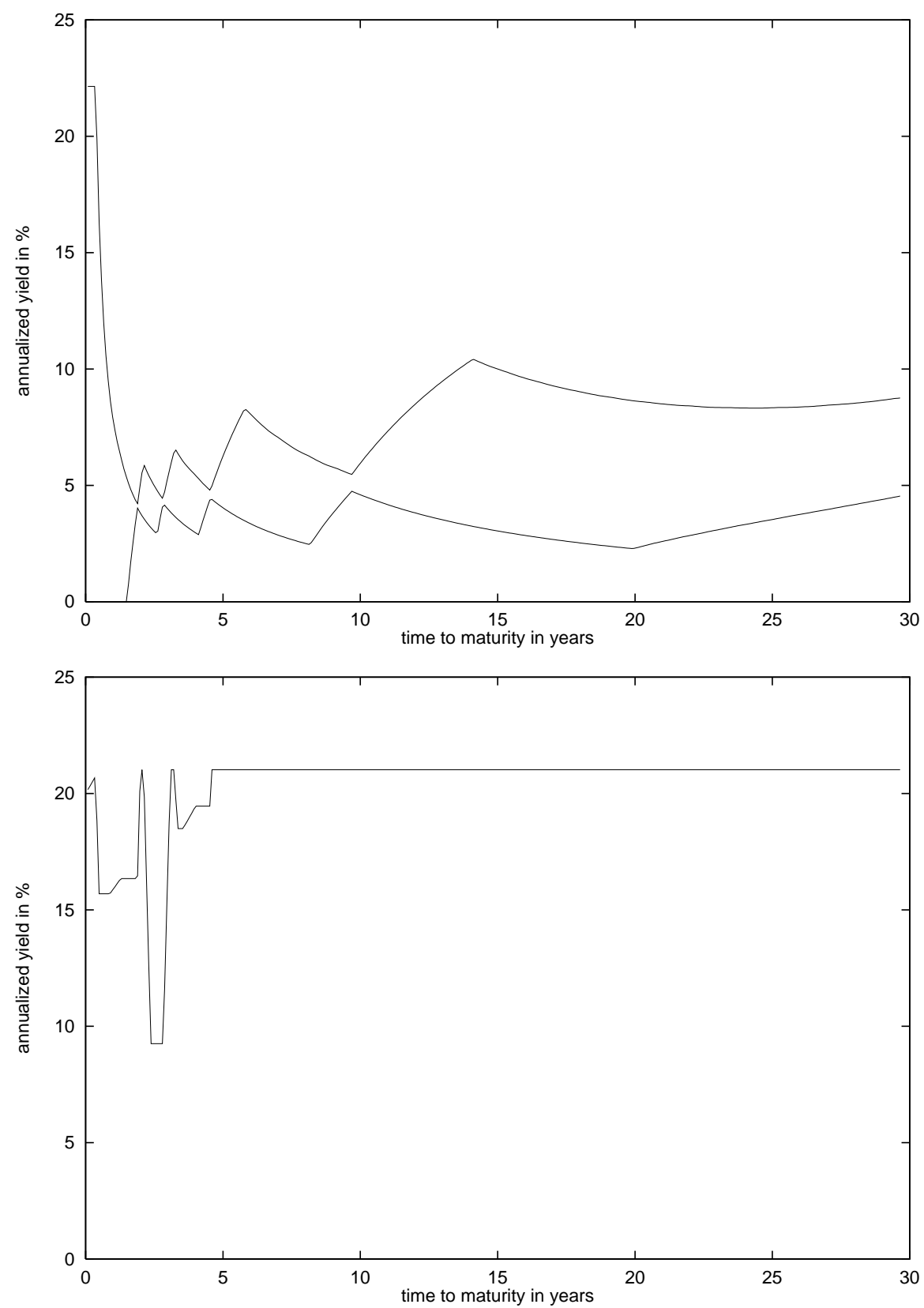

Figure 3: Good Deal Bounds for the Term Structure under First Order Conditions

The upper graph shows the good deal bounds for the zero-bond yield curve. The lower graph shows the bounds for the 6 -month forward rate curve. The bounds are computed w.r.t. the 5 benchmark bonds and the first order conditions with $a^{(1)}=0, b^{(1)}=20 \%$. The lower bounds for the forward rate curve is zero. $b^{(1)}=20 \%$ means $20 \%$ continuously compounded rate whereas the graphs always show annualized rates. This explains why the upper bound is above $20 \%$. 

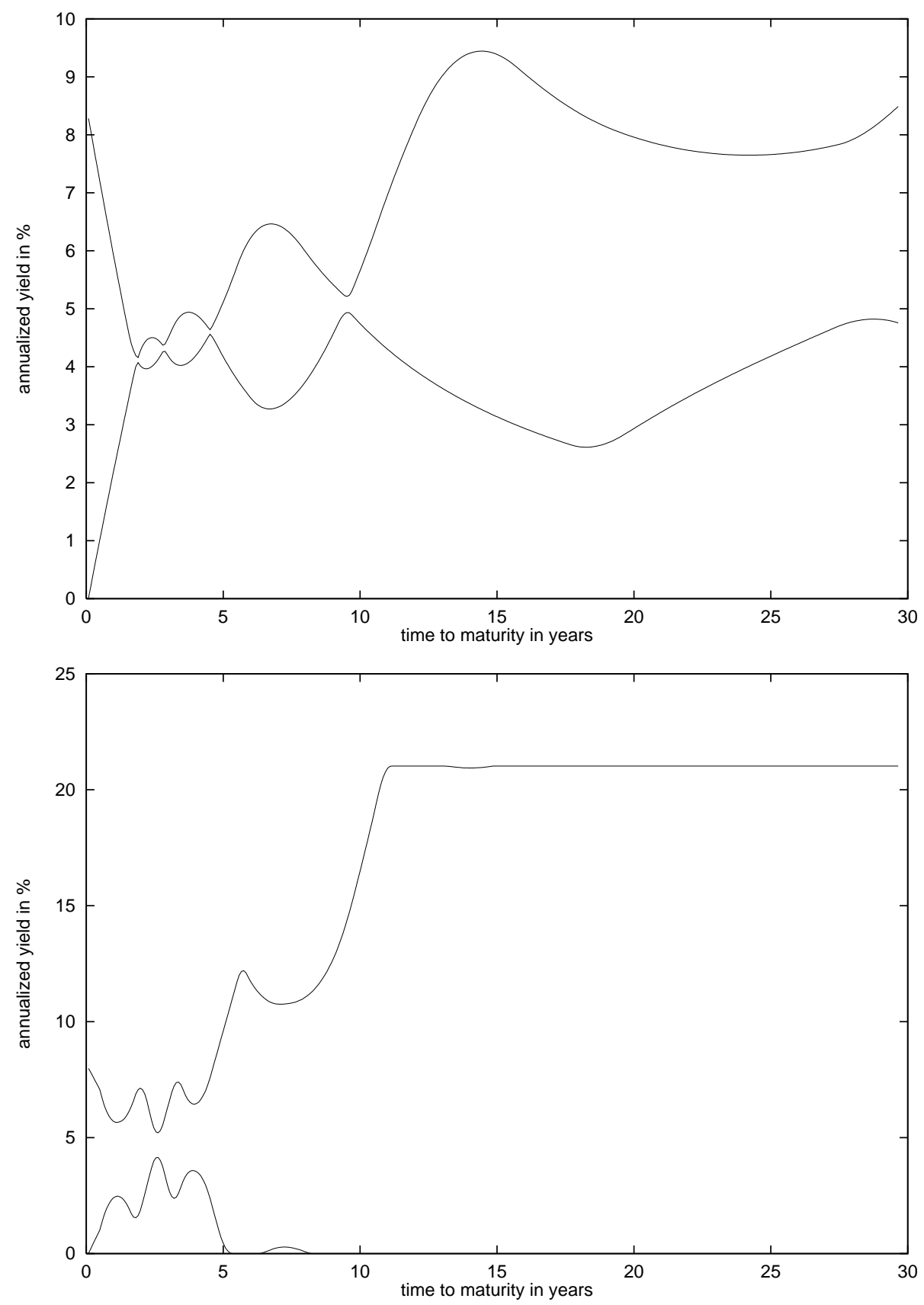

Figure 4: Good Deal Bounds for the Term Structure under Second Order Conditions

The upper graph shows the good deal bounds for the zero-bond yield curve. The lower graph shows the bounds for the 6 -month forward rate curve. The bounds are computed w.r.t. the 5 benchmark bonds and the first and second order conditions with $a^{(1)}=0, b^{(1)}=20 \%$, $a^{(2)}=4 \%, b^{(2)}=4 \%$. 

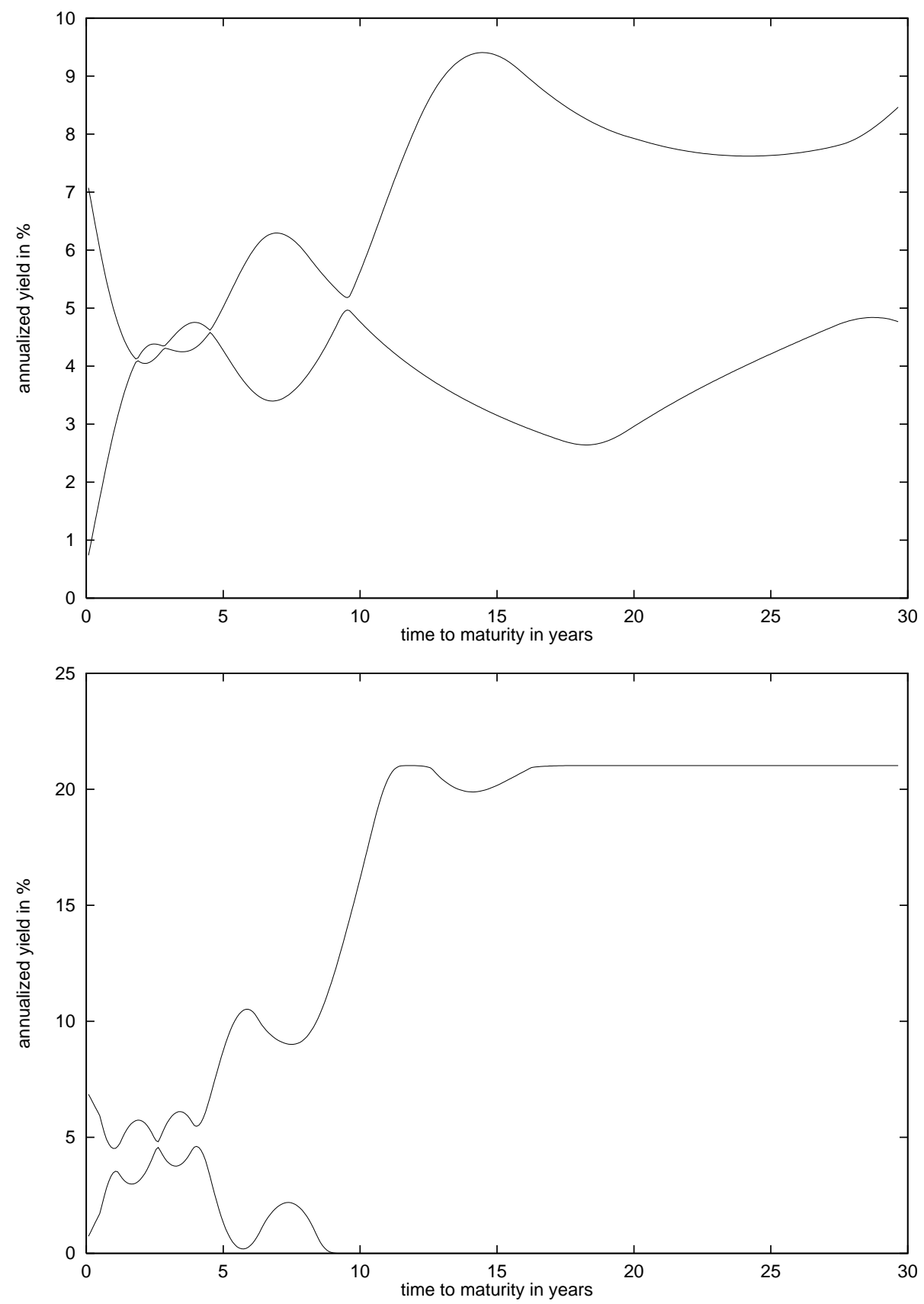

Figure 5: Good Deal Bounds for the Term Structure under Third Order Conditions

The upper graph shows the good deal bounds for the zero-bond yield curve. The lower graph shows the bounds for the 6-month forward rate curve. The bounds are computed w.r.t. the 5 benchmark bonds and the first, second, and third order conditions with $a^{(1)}=0, b^{(1)}=20 \%$, $a^{(2)}=4 \%, b^{(2)}=4 \%, a^{(3)}=4 \%$, and $b^{(3)}=4 \%$. 

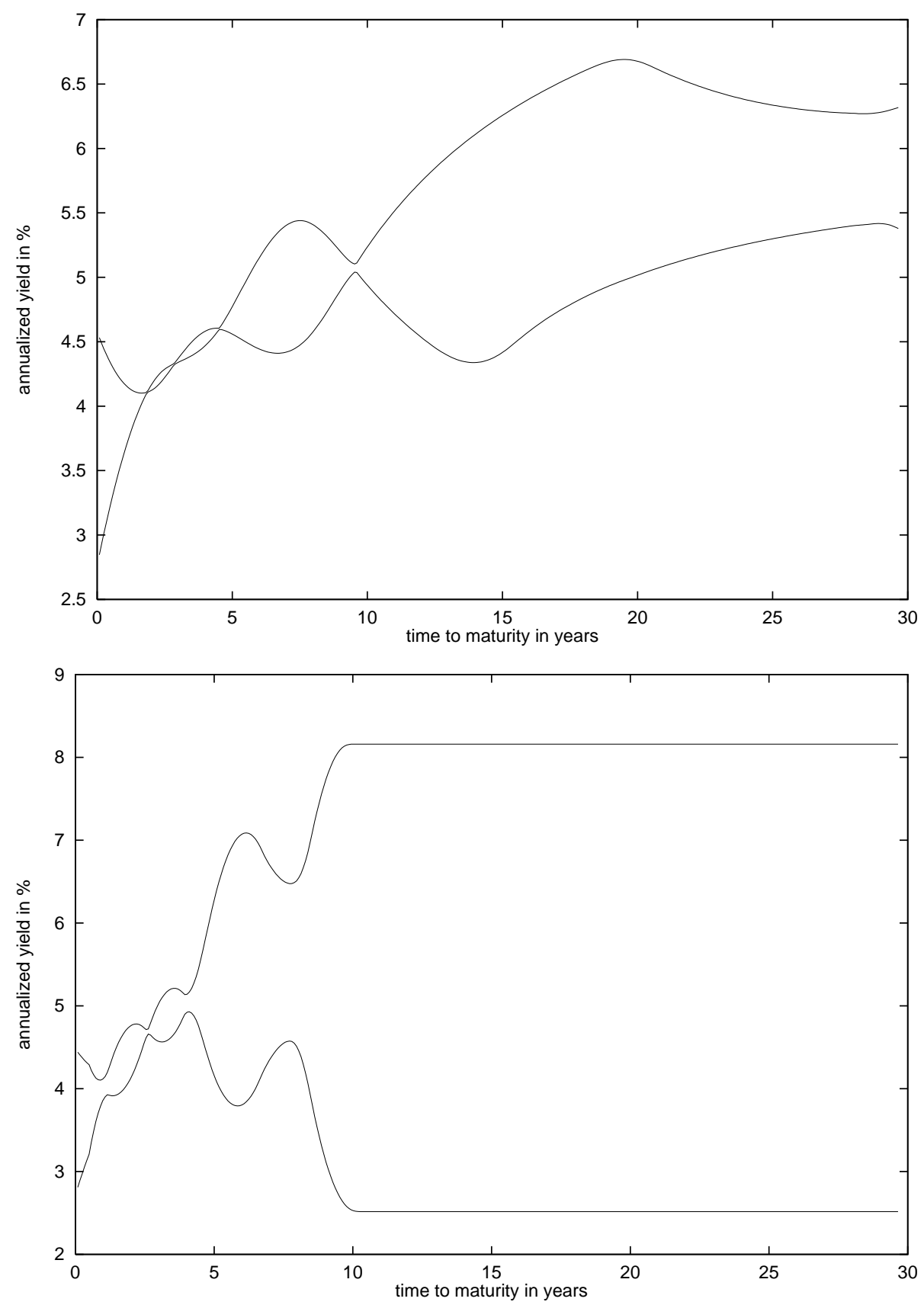

Figure 6: Good Deal Bounds for the Term Structure under Third Order Conditions

The upper graph shows the good deal bounds for the zero-bond yield curve. The lower graph shows the bounds for the 6-month forward rate curve. The bounds are computed w.r.t. the 5 benchmark bonds and the first, second, and third order conditions with $a^{(1)}=2.5 \%$, $b^{(1)}=8 \%, a^{(2)}=2 \%, b^{(2)}=2 \%, a^{(3)}=1 \%$, and $b^{(3)}=1 \%$. 
set $a^{(2)}$ rather small:

$$
a_{s}^{(2)}=0.1 \% \cdot 2^{-s / 10} .
$$

$a^{(3)}=0.1 \%$ would mean that we allow the forward rate curve to tilt from its $f^{\prime}(0) \approx 0.5 \%$ at the short end to $f^{\prime}(5)=0$ at 5 years time to maturity. This would be a rather high curvature, so

$$
a_{s}^{(3)}=0.1 \% \cdot 2^{-s / 10}
$$

cannot be considered overly restrictive. Since it looks as if the forward rate curve is concave, we set $b^{(3)}$ rather low:

$$
b_{s}^{(3)}=0.01 \% \cdot 2^{-s / 10}
$$

The resulting bounds for the term structure are shown in figure 7 .

\subsection{Identification of Tax Clientele Effects}

If capital gains and coupons are taxed differently and investors with different tax rates exist, then there may be tax clientele effects in the sense that certain securities are rationally held only by investors of certain tax classes. In order to show that a bond is definitely not held by investors of a specific tax class $k$, Schaefer (1982) computes the upper arbitrage bound of its class- $k$ after-tax cash stream $\bar{\pi}\left(-S_{i}^{k}, C_{i 1}^{k}, \ldots, C_{i n}^{k}\right)$

$$
=\min _{p, x}\left\{p \mid\left(\begin{array}{cccc}
1 & 0 & \ldots & 0 \\
& C &
\end{array}\right)^{\prime}\left(\begin{array}{l}
p \\
x
\end{array}\right) \succeq_{a}\left(\begin{array}{c}
-S_{i}^{k} \\
C_{i 1}^{k} \\
\ldots \\
C_{i n}^{k}
\end{array}\right), x \geq 0, p \in \mathbf{R}\right\}^{11}
$$

If that value is negative, the bond is called dominated for class $k$. Short-selling is assumed to be infinitely expensive, that is, the cash flow matrix contains only the "long" after-tax cash streams: $C^{k}=\left(-S^{k} \mid P^{k}\right)$.

It was pointed out especially by Rasch (1996) that the above method is asymmetric in its ability to detect tax clienteles. Bonds with high coupons can be very well super-replicated by a series of low-coupon bonds with decreasing time to maturity, in the sense that the two cash streams in the $\succeq_{a}$-comparison are almost the same. In order to super-replicate a bond with a very low coupon, however, one has to match its principal payment with the principal payment of a bond with a higher coupon. The higher the coupon of the superreplicating bond, the more is "lost" in the $\succeq_{a}$-comparison. Note that this is not a mathematical fact, but a heuristic explanation why in practice most least-cost super-replicating portfolios are of the following structure:

\footnotetext{
$0\}$.

${ }^{11} \succeq_{a}$ is the arbitrage dominance relation corresponding to $K_{a}=\left\{u \mid u_{1} \geq u_{2} \geq \ldots \geq u_{n} \geq\right.$
} 

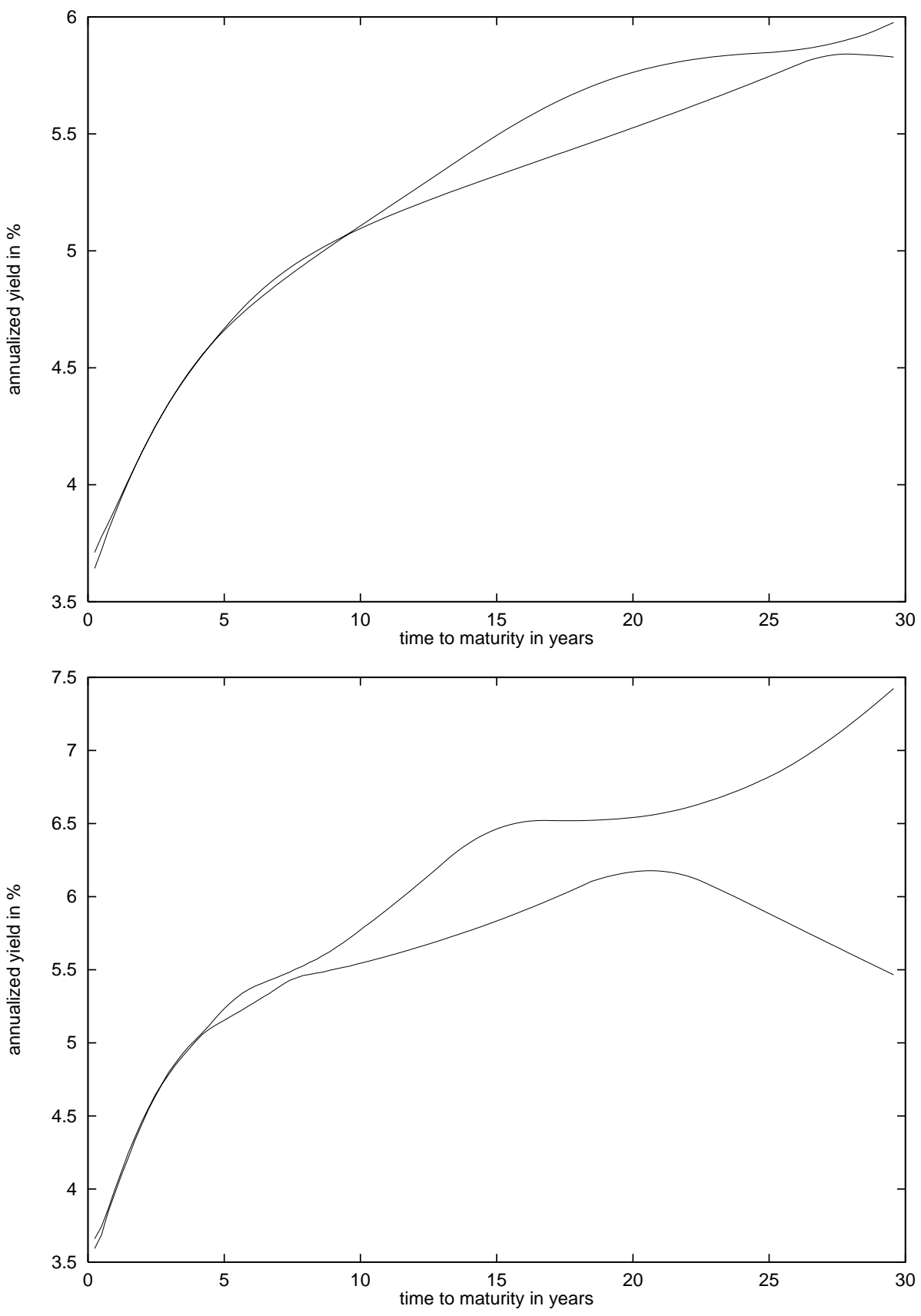

Figure 7: Good Deal Bounds for the Term Structure under Time Dependent Third Order Conditions

The upper graph shows the good deal bounds for the zero-bond yield curve. The lower graph shows the bounds for the 6 -month forward rate curve. The bounds are computed w.r.t. the 5 benchmark bonds, the first order conditions, and the time-dependent second and third order conditions. 
- All bonds in the super-replicating portfolio have the same or a shorter time to maturity than the dominated bond.

- The bond in the super-replicating portfolio that "provides the principal payment" has the same or a lower coupon than the dominated bond.

This limits the effectiveness of the standard arbitrage approach in finding bonds that are overvalued in a common sense of the word (from the viewpoint of a specific tax class).

Bond 110083 in August 1981 provides an example for a bond that is significantly overvalued (from a tax-free viewpoint) under moderate first and second order conditions, but is not dominated in Schaefer's sense. The following table shows how much more expensive than its least-cost super-replicating portfolio the bond 110083 was on August 28, 1981, under increasingly stricter conditions on the admissible term structures:

\begin{tabular}{|l|r|l|} 
dominance relation & $-\bar{\pi}(110083)$ & $\begin{array}{l}\text { bonds in the super- } \\
\text { replicating portfolio }\end{array}$ \\
\hline arbitrage & 0 & 110083 \\
1.order: $a^{(1)}=0, b^{(1)}=20 \%$ & $1.63 \%$ & 113401 \\
1. \& 2.order: $a^{(2)}=b^{(2)}=4 \%$ & $3.11 \%$ & 113401,113402 \\
$1 ., 2 . \&$ 3.order: $a^{(3)}=b^{(3)}=4 \%$ & $3.12 \%$ & 113401,113402
\end{tabular}

The cash flow matrix includes all German government bonds except Post and Bahn bonds that were traded at the Frankfurt stock exchange on that day.

\begin{tabular}{|c|c|r|r|r|} 
WKN $^{12}$ & maturity date & coupon & issue volume & issue year \\
\hline 110100 & $01 / 01 / 90$ & 7.75 & 1500 & 80 \\
113400 & $04 / 01 / 90$ & 10.00 & 1100 & 80 \\
110083 & $05 / 01 / 90$ & 5.75 & 500 & 78 \\
113401 & $07 / 01 / 90$ & 8.25 & 1500 & 80 \\
113402 & $11 / 01 / 90$ & 8.25 & 1500 & 80
\end{tabular}

Table 2: Parameters of Bonds 110100, 113400, 110083, 113401, and 113402

The overvaluation of bond 110083 from a tax-free viewpoint is very plausibly explained by tax effects, since it is the only low-coupon bond with a maturity of about 9 years. (See table 2.) Although the overvaluation compared to 113401 and 113402 is significant, this is not detected by the arbitrage dominance relation. There are three reasons for this. First, 113401 and 113402 mature later than 110083, consequently they are no good candidates for the super-replicating portfolio. 113400 has an extremely high coupon, thus neither 113400 is a good candidate. Third, 113400 is itself a bit overvalued. Under 
third order conditions, 113400 is dominated by $0.87 \%$ of face value. Its superreplicating portfolio contains 113401 and 110100 as well as some bonds with shorter time to maturity in lower quantities. ${ }^{13}$

\section{Conclusion}

The linear higher order conditions on the term structure allow to compute valuation bounds for any deterministic cash stream. The higher order conditions have 2 economic and 1 "graphic" interpretation:

1. Future short-term interest rate movements are restricted.

2. Certain auxiliary cash streams (higher order FRAs) are available.

3. The lower order derivatives of the discount function are bounded. This is approximately the condition that the forward rate curve and its (lower order) derivatives are bounded.

Starting from the easily interpretable bounds on the forward rate curve and its derivatives, which are nonlinear in the discount factors, we derive linear conditions that are only slightly less restrictive than the nonlinear conditions. (The term structure packet defined by the nonlinear conditions is contained in the term structure packet defined by the linear conditions, but the difference is small.) The linearization of the term structure constraints has two advantages. First, the valuation bounds can be computed by the highly developed LP solvers. Second, the constraints have an economic meaning as auxiliary cash streams. Thus, price discrepancies can be easily translated into trading strategies.

The higher order conditions on the term structure are related to extremal event statistics of short-term interest rates. This puts the resulting valuation bounds conceptually (but not mathematically) close to methods like value at risk.

Depending on the choice of the constraints on the forward rate curve the valuation bounds can be very wide or very close. Arbitrage bounds are contained as a special case. On the other end of the extreme, the valuation bounds on the term structure itself behave like quadratic splines in the forward rate curve if the third order parameters are chosen very small.

Statistical methods that derive a single price system from observed prices abound. These can be complemented by methods that compute valuation bounds. Valuation bounds that are free of parametric and distributional assumptions have an advantage over statistical methods when one suspects that a certain regression equation - for example the present value equation - or a

\footnotetext{
${ }^{13}$ According to a bond dealer, the overvaluation of 113400 could be due to the fact that financial institutions sometimes use bonds with a very high coupon for window dressing purposes in their accounting.
} 
certain method - like smoothing splines (Fisher et al.; 1995) - are not suitable in a specific market. Schaefer's (1982) work is a prominent example of the use of arbitrage bounds for proving that the assumption that all bonds are priced according to the present value equation under a single term structure is wrong. The ability of the arbitrage dominance principle to detect price discrepancies in bond markets, is however, limited. The valuation bounds under the linear higher order conditions on the term structure generalize arbitrage bounds and provide a sharper method when the arbitrage principle is too weak.

\section{Proofs}

Proof of Proposition 2. $\frac{\partial}{\partial r_{1}} v>0$, which implies that a local extremum cannot be attained in the interior of the feasible set and not in the relative interior of the edges defined by $\left\{r_{2}=a^{(1)}\right\}$ and $\left\{r_{2}=b^{(1)}\right\}$. (17) ensures $\frac{\partial}{\partial r_{2}} v<0$, which implies that an extremum cannot be attained in the relative interior of the edges $\left\{r_{1}=a^{(1)}\right\}$ and $\left\{r_{1}=b^{(1)}\right\}$ either. After sorting out $\left(r_{1}, r_{2}\right)=\left(b^{(1)}, b^{(1)}\right)$ and $\left(r_{1}, r_{2}\right)=\left(a^{(1)}, a^{(1)}\right)$ as being suboptimal, the maximum is attained somewhere on the edge defined by $\left\{r_{2}-r_{1}=-a^{(2)} \Delta\right\}$ and the minimum is attained somewhere on the edge defined by $\left\{r_{2}-r_{1}=b^{(2)} \Delta\right\}$. (18) and (21) ensure that these edges exist (that the relative interior of the feasible set w.r.t. the lines defined by these boundary conditions is nonempty), and the points $\left(r_{1}, r_{2}\right)=\left(b^{(1)}, a^{(1)}\right)$ and $\left(r_{1}, r_{2}\right)=\left(a^{(1)}, b^{(1)}\right)$ are infeasible.

The directional second derivative of $v$ on the line $\left\{r_{2}-r_{1}=\right.$ const. $\}$ is positive, so the maximum is attained at one of the endpoints (a) or (b), and the minimum is attained at the point $(\mathrm{d})$, where the directional first derivative is zero, or at one of the endpoints (c), (e).

Proof of Proposition 3. The proof consists in going through all combinations of which of the boundary conditions (excluding $\left\{g\left(r_{1}, r_{2}, r_{3}\right)=-a^{(3)}\right\}$ and $\left.\left\{g\left(r_{1}, r_{2}, r_{3}\right)=b^{(3)}\right\}\right)$ are binding and showing that either the corresponding subspace has no point in common with the feasible set or one of the necessary conditions for a local extremum does not hold.

Conditions (17) and (26) ensure that $\frac{\partial}{\partial r_{1}} v>0, \frac{\partial}{\partial r_{2}} v<0$, and $\frac{\partial}{\partial r_{3}} v>0$. This implies that there cannot be a local extremum in

- the interior of the feasible set,

- the relative interior of the faces $\left\{r_{i}=\right.$ const. $\}$,

- the relative interior of the faces $\left\{r_{3}-r_{2}=\right.$ const. $\}\left(\frac{\partial}{\partial r_{1}} v>0\right)$,

- the relative interior of the faces $\left\{r_{2}-r_{1}=\right.$ const. $\}\left(\frac{\partial}{\partial r_{3}} v>0\right)$.

This excludes the relative interior of all faces except $\left\{g\left(r_{1}, r_{2}, r_{3}\right)=\right.$ const. $\}$. 


\begin{tabular}{|c|c|c|}
\hline subspace & increase & decrease \\
\hline$\left\{r_{1}=\right.$ const.,$r_{3}=$ const. $\}$ & $(0,-1,0)$ & $(0,1,0)$ \\
\hline$\left\{r_{2}=c_{1}, r_{i}=c_{2}\right\}_{i=1,3 ; c_{1} \neq c_{2}}$ & infeasible: (27), & $,(29),(30)$ \\
\hline$\left\{r_{2}=c, r_{1}=c\right\}$ & $(0,0,1)$ & $(0,0,-1)$ \\
\hline$\left\{r_{2}=c, r_{3}=c\right\}$ & $(1,0,0)$ & $(-1,0,0)$ \\
\hline$\left\{r_{3}-r_{2}=c_{1}, r_{2}-r_{1}=c_{2}\right\}_{c_{1} \neq c_{2}}$ & infeasible: (31), & \\
\hline$\left\{r_{3}-r_{2}=-\Delta_{23} a^{(2)}, r_{2}-r_{1}=-\Delta_{12} a^{(2)}\right\}$ & $(0,0,1)$ & $(-1,0,0)$ \\
\hline$\left\{r_{3}-r_{2}=\Delta_{23} b^{(2)}, r_{2}-r_{1}=\Delta_{12} b^{(2)}\right\}$ & $(1,0,0)$ & $(0,0,-1)$ \\
\hline$\left\{r_{i}=\text { const., } r_{2}-r_{1}=\text { const. }\right\}_{i=1,2}$ & $(0,0,1)$ & $(0,0,-1)$ \\
\hline$\left\{r_{i}=\text { const., } r_{3}-r_{2}=\text { const. }\right\}_{i=2,3}$ & $(1,0,0)$ & $(-1,0,0)$ \\
\hline$\left\{r_{3}=a^{(1)}, r_{2}-r_{1}=\Delta_{12} b^{(2)}\right\}$ & infeasible: $(35)$ & \\
\hline$\left\{r_{3}=b^{(1)}, r_{2}-r_{1}=\Delta_{12} b^{(2)}\right\}$ & $(1,0,0)$ & $(0,0,-1)$ \\
\hline$\left\{r_{3}=b^{(1)}, r_{2}-r_{1}=-\Delta_{12} a^{(2)}\right\}$ & infeasible: $(33)$ & \\
\hline$\left\{r_{3}=a^{(1)}, r_{2}-r_{1}=-\Delta_{12} a^{(2)}\right\}$ & $(0,0,-1)$ & $(-1,0,0)$ \\
\hline$\left\{r_{1}=b^{(1)}, r_{3}-r_{2}=\Delta_{23} b^{(2)}\right\}$ & infeasible: $(34)$ & \\
\hline$\left\{r_{1}=a^{(1)}, r_{3}-r_{2}=\Delta_{23} b^{(2)}\right\}$ & $(1,0,0)$ & $(0,0,-1)$ \\
\hline$\left\{r_{1}=a^{(1)}, r_{3}-r_{2}=-\Delta_{23} a^{(2)}\right\}$ & infeasible: $(36)$ & \\
\hline$\left\{r_{1}=b^{(1)}, r_{3}-r_{2}=-\Delta_{23} a^{(2)}\right\}$ & $(0,0,1)$ & $(-1,0,0)$ \\
\hline
\end{tabular}

\section{Table 3: One-Dimensional Subspaces}

The first column defines one or more 1-dimensional subspaces. The other columns contain either directions that improve the objective function or a reason why these lines have no point in common with the feasible set.

Table 3 covers all points in the relative interior of the edges. The first column defines one or more 1-dimensional subspaces. The other columns contain either directions that improve the objective function or a reason why these lines have no point in common with the feasible set.

From $\frac{\partial}{\partial r_{1}} v>0, \frac{\partial}{\partial r_{2}} v<0$, and $\frac{\partial}{\partial r_{3}} v>0$ follows that only the following conditions can be binding at a local maximum:

$$
\begin{array}{lll}
\left(r_{1}=b^{(1)}\right. & \text { or } & \left.r_{2}-r_{1}=-\Delta_{12} a^{(2)}\right) \quad \text { and } \\
\left(r_{2}=a^{(1)}\right. & \text { or } & \left.r_{2}-r_{1}=-\Delta_{12} a^{(2)} \quad \text { or } \quad r_{3}-r_{2}=\Delta_{23} b^{(2)}\right) \quad \text { and } \\
\left(r_{3}=b^{(1)}\right. & \text { or } & \left.r_{3}-r_{2}=\Delta_{23} b^{(2)}\right) .
\end{array}
$$

The resulting points are listed in table 4 and shown to be infeasible.

From $\frac{\partial}{\partial r_{1}} v>0, \frac{\partial}{\partial r_{2}} v<0$, and $\frac{\partial}{\partial r_{3}} v>0$ follows that only the following conditions can be binding at a local minimum:

$$
\left(r_{1}=a^{(1)} \quad \text { or } \quad r_{2}-r_{1}=\Delta_{12} b^{(2)}\right) \quad \text { and }
$$




\begin{tabular}{|lll|l|}
\hline \multicolumn{3}{|c|}{ defining boundaries } & cond. \\
\hline$r_{1}=b^{(1)}$ & $r_{2}=a^{(1)}$ & any other & $(28)$ \\
$r_{1}=b^{(1)}$ & $r_{2}-r_{1}=-\Delta_{12} a^{(2)}$ & $r_{3}=b^{(1)}$ & $(33)$ \\
$r_{1}=b^{(1)}$ & $r_{2}-r_{1}=-\Delta_{12} a^{(2)}$ & $r_{3}-r_{2}=\Delta_{23} b^{(2)}$ & $(31)$ \\
$r_{1}=b^{(1)}$ & $r_{3}-r_{2}=\Delta_{23} b^{(2)}$ & $r_{3}=b^{(1)}$ & $(34)$ \\
$r_{2}-r_{1}=-\Delta_{12} a^{(2)}$ & $r_{2}=a^{(1)}$ & $r_{3}=b^{(1)}$ & $(27)$ \\
$r_{2}-r_{1}=-\Delta_{12} a^{(2)}$ & $r_{2}=a^{(1)}$ & $r_{3}-r_{2}=\Delta_{23} b^{(2)}$ & $(31)$ \\
$r_{2}-r_{1}=-\Delta_{12} a^{(2)}$ & $r_{3}-r_{2}=\Delta_{23} b^{(2)}$ & $r_{3}=b^{(1)}$ & $(31)$ \\
\hline
\end{tabular}

Table 4: Candidates for a Maximum

The last column shows the reason why the point defined by the three boundary conditions is infeasible.

\begin{tabular}{|lll|l|}
\hline \multicolumn{3}{|c|}{ defining boundaries } & cond. \\
\hline$r_{1}=a^{(1)}$ & $r_{2}=b^{(1)}$ & any other & $(30)$ \\
$r_{1}=a^{(1)}$ & $r_{2}-r_{1}=\Delta_{12} b^{(2)}$ & $r_{3}=a^{(1)}$ & $(35)$ \\
$r_{1}=a^{(1)}$ & $r_{2}-r_{1}=\Delta_{12} b^{(2)}$ & $r_{3}-r_{2}=-\Delta_{23} a^{(2)}$ & $(32)$ \\
$r_{1}=a^{(1)}$ & $r_{3}-r_{2}=-\Delta_{23} a^{(2)}$ & $r_{3}=a^{(1)}$ & $(36)$ \\
$r_{2}-r_{1}=\Delta_{12} b^{(2)}$ & $r_{2}=b^{(1)}$ & $r_{3}=a^{(1)}$ & $(29)$ \\
$r_{2}-r_{1}=\Delta_{12} b^{(2)}$ & $r_{2}=b^{(1)}$ & $r_{3}-r_{2}=-\Delta_{23} a^{(2)}$ & $(32)$ \\
$r_{2}-r_{1}=\Delta_{12} b^{(2)}$ & $r_{3}-r_{2}=-\Delta_{23} a^{(2)}$ & $r_{3}=a^{(1)}$ & $(32)$ \\
\hline
\end{tabular}

\section{Table 5: Candidates for a Minimum}

The last column shows the reason why the point defined by the three boundary conditions is infeasible.

$$
\begin{array}{lll}
\left(r_{2}=b^{(1)}\right. & \text { or } & \left.r_{2}-r_{1}=\Delta_{12} b^{(2)} \quad \text { or } \quad r_{3}-r_{2}=-\Delta_{23} a^{(2)}\right) \quad \text { and } \\
\left(r_{3}=a^{(1)}\right. & \text { or } & \left.r_{3}-r_{2}=-\Delta_{23} a^{(2)}\right) .
\end{array}
$$

The resulting points are listed in table 5 and shown to be infeasible.

We have now proved that one of the conditions $\left\{g\left(r_{1}, r_{2}, r_{3}\right)=\right.$ const. $\}$ is binding at a local extremum. Taking the direction $(1,-1,1)$, it is seen that the maximum can only be attained on $\left\{g\left(r_{1}, r_{2}, r_{3}\right)=b^{(3)}\right\}$ and the minimum on $\left\{g\left(r_{1}, r_{2}, r_{3}\right)=-a^{(3)}\right\}$.

Proof of Proposition 4. On the face $\left\{g\left(r_{1}, r_{2}, r_{3}\right)=c\right\}$, we substitute $r_{1}$ by

$$
r_{1}=\frac{1}{\Delta_{23}}\left(\Delta_{123} \Delta_{12} \Delta_{23} c+\left(\Delta_{12}+\Delta_{23}\right) r_{2}-\Delta_{12} r_{3}\right)
$$

to get a function $\tilde{v}\left(r_{2}, r_{3}\right)=v\left(r_{1}, r_{2}, r_{3}\right)$. With

$$
F_{1}:=e^{\Delta_{1} r_{1}+\Delta_{2} r_{2}+\Delta_{3} r_{3}}
$$




$$
\begin{aligned}
& F_{2}:=e^{\Delta_{2} r_{2}+\Delta_{3} r_{3}}, \quad \text { and } \\
& F_{3}:=e^{\Delta_{3} r_{3}}
\end{aligned}
$$

we get

$$
\begin{aligned}
\frac{\partial}{\partial r_{2}} \tilde{v} & =A F_{1}+\Delta_{2} F_{2} z_{2} \text { and } \\
\frac{\partial}{\partial r_{3}} \tilde{v} & =B F_{1}+\Delta_{3}\left(F_{2} z_{2}+F_{3} z_{3}\right) .
\end{aligned}
$$

With $x:=F_{2} z_{2} / F_{1}, y:=F_{3} z_{3} / F_{1}$, the first order condition $\nabla \tilde{v}=0$ can be written as

$$
\begin{aligned}
x & =-A / \Delta_{2}, \\
y+x & =-B / \Delta_{3},
\end{aligned}
$$

which has a unique solution in $(x, y)$. Since (17) and (26) imply $x+1<0$ and $x+y+1>0$, respectively, this corresponds to a unique solution in $\left(r_{2}, r_{3}\right)$ (for fixed $\left.\left(z_{2}, z_{3}\right)\right)$.

The second derivatives are:

$$
\begin{aligned}
\frac{\partial^{2}}{\partial r_{2}^{2}} \tilde{v} & =A^{2} F_{1}+\Delta_{2}^{2} F_{2} z_{2} \\
\frac{\partial^{2}}{\partial r_{3}^{2}} \tilde{v} & =B^{2} F_{1}+\Delta_{3}^{2}\left(F_{2} z_{2}+F_{3} z_{3}\right) \\
\frac{\partial^{2}}{\partial r_{2} \partial r_{3}} \tilde{v} & =A B F_{1}+\Delta_{2} \Delta_{3} F_{2} z_{2}
\end{aligned}
$$

A sufficient condition for the Hessian being negative definite is $\frac{\partial^{2}}{\partial r_{2}^{2}} \tilde{v} \frac{\partial^{2}}{\partial r_{3}^{2}} \tilde{v}-$ $\left(\frac{\partial^{2}}{\partial r_{2} \partial r_{3}} \tilde{v}\right)^{2}<0$. This condition can be simplified to

$$
y \Delta_{3}^{2}\left(A^{2}+\Delta_{2}^{2} x\right)+x\left(A \Delta_{3}-B \Delta_{2}\right)^{2}<0 .
$$

At the local extremum defined by $\nabla \tilde{v}=0$, the condition simplifies to

$$
x y(x+y+1)<0 .
$$

We see that the Hessian is always negative definite at the local extremum, but maybe not for all values of $\left(z_{2}, z_{3}\right)$ under the conditions $(17),(26)$. So we need the additional condition (37).

That $\tilde{v}$ is strictly concave ensures that the minimum can only be attained at extremal points (corners) of the simplex defined by (9), (10), and (11). From $\frac{\partial}{\partial r_{1}} v>0, \frac{\partial}{\partial r_{2}} v<0$, and $\frac{\partial}{\partial r_{3}} v>0$ follows that only the following conditions can be binding at a local minimum:

$$
\begin{gathered}
\left(r_{1}=a^{(1)} \quad \text { or } \quad r_{2}-r_{1}=\Delta_{12} b^{(2)} \quad \text { or } \quad g\left(r_{1}, r_{2}, r_{3}\right)=-\Delta_{123} a^{(3)}\right) \\
\quad \text { and }
\end{gathered}
$$




\begin{tabular}{|c|c|c|}
\hline \multicolumn{2}{|c|}{ defining boundaries } & feasible? \\
\hline$r_{2}=b_{1}$ & $r_{3}=a_{1}$ & (29) \\
\hline$r_{2}=b_{1}$ & $r_{3}-r_{2}=-\Delta_{23} a^{(2)}$ & $*$ \\
\hline$r_{2}-r_{1}=\Delta_{12} b^{(2)}$ & $r_{3}=a_{1}$ & $(35)$ \\
\hline$r_{2}-r_{1}=\Delta_{12} b^{(2)}$ & $r_{3}-r_{2}=-\Delta_{23} a^{(2)}$ & $(32)$ \\
\hline$r_{3}-r_{2}=-\Delta_{23} a^{(2)}$ & $r_{3}=a_{1}$ & $*$ \\
\hline$r_{1}=a_{1}$ & $r_{3}=a_{1}$ & $*$ \\
\hline$r_{1}=a_{1}$ & $r_{3}-r_{2}=-\Delta_{23} a^{(2)}$ & $(36)$ \\
\hline$r_{1}=a_{1}$ & $r_{2}=b^{(1)}$ & $(36)$ \\
\hline$r_{1}=a_{1}$ & $r_{2}-r_{1}=\Delta_{12} b^{(2)}$ & $*$ \\
\hline$r_{2}-r_{1}=\Delta_{12} b^{(2)}$ & $r_{2}=b^{(1)}$ & $*$ \\
\hline
\end{tabular}

Table 6: Candidates for a Minimum

The points are defined by the two conditions shown and $g\left(r_{1}, r_{2}, r_{3}\right)=-\Delta_{123} a^{(3)}$. The last column shows the reason why the point is infeasible or a star when the point remains as a candidate for a minimum.

$$
\begin{aligned}
& \left(r_{2}=b^{(1)} \quad \text { or } \quad r_{2}-r_{1}=\Delta_{12} b^{(2)}\right. \\
& \text { or } \left.\left.\quad r_{3}-r_{2}=-\Delta_{23} a^{(2)} \quad \text { or } \quad g\left(r_{1}, r_{2}, r_{3}\right)=-\Delta_{123} a^{(3)}\right)\right) \\
& \text { and } \\
& \left.\left(r_{3}=a^{(1)} \quad \text { or } \quad r_{3}-r_{2}=-\Delta_{23} a^{(2)} \quad \text { or } \quad g\left(r_{1}, r_{2}, r_{3}\right)=-\Delta_{123} a^{(3)}\right)\right) .
\end{aligned}
$$

The resulting points are listed in table 6 .

\section{References}

Anderson, N., Breedon, F., Deacon, M., Derry, A. and Murphy, G. (1996). Estimating and Interpreting the Yield Curve, Series in Financial Economics and Quantitative Analysis, John Wiley.

Artzner, P., Delbaen, F., Eber, J.-M. and Heath, D. (1998). Coherent measures of risk, Université Louis Pasteur, Strasbourg. Working paper in several versions since 1996.

Bekdache, B. and Baum, C. F. (1994). Comparing alternative models of the term structure of interest rates, Working Paper No. 271, Boston College.

Bertsekas, D. P. (1995). Dynamic Programming and Optimal Control, Athena Scientific, Belmont, Massachusetts. 
Chambers, D. R., Carleton, W. T. and Waldman, D. W. (1984). A new approach to estimation of the term structure of interest rates, Journal of Financial and Quantitative Analysis 19(3): 233-252.

Coleman, T. S., Fisher, L. and Ibbotson, R. G. (1992). Estimating the term structure of interest rates from data that include the prices of coupon bonds, Journal of Fixed Income 2: 85-116.

Dermody, J. C. and Rockafellar, R. T. (1991). Cash stream valuation in the face of transaction costs and taxes, Mathematical Finance 1(1): 31-54.

Dermody, J. C. and Rockafellar, R. T. (1995). Tax basis and nonlinearity in cash stream valuation, Mathematical Finance 5(2): 97-119.

Dybvig, P. and Ross, S. (1986). Tax clienteles and asset pricing, Journal of Finance $\mathbf{X L I}(3)$ : 751-763.

Fisher, M., Nychka, D. and Zervos, D. (1995). Fitting the term structure of interest rates with smoothing splines. Finance and Economics Discussion Series, 95-1.

Hodges, S. D. and Schaefer, S. M. (1977). A model for bond portfolio improvement, Journal of Financial and Quantitative Analysis 12: 243-260.

Ingersoll, J. E. (1987). Theory of Financial Decision Making, Studies in Financial Economics, Rowman \& Littlefield Publishers, Inc., Savage, Maryland.

Jaschke, S. R. (1998). Arbitrage bounds for the term structure of interest rates, Finance and Stochastics 2(1): 29-40.

McCulloch, H. (1971). Measuring the term structure of interest rates, Journal of Business 44(1): 19-31.

McCulloch, H. (1975). The tax-adjusted yield curve, Journal of Finance 30(3): 811-830.

Pichler, S. (1995). Ermittlung der Zinsstruktur, Deutscher Universitätsverlag, Gabler Verlag, Wiesbaden.

Rasch, S. (1996). Steuer-Klientel-Effekte an DM-Anleihemärkten, Vol. 14 of Schriftenreihe des ZEW, Nomos Verlagsgesellschaft, Baden-Baden.

Ronn, E. I. (1987). A new linear programming approach to bond portfolio management, Journal of Financial and Quantitative Analysis 22(4): 439466 .

Schaefer, S. (1981). Measuring a tax-specific term structure of interest rates in the market for British government securities, Economic Journal 91: 415-38. 
Schaefer, S. M. (1982). Tax-induced clientele effects in the market for British government securities, Journal of Financial Economics 10: 121-159.

Schich, S. T. (1996). Alternative Spezifikationen der deutschen Zinsstrukturkurve und ihr Informationsgehalt hinsichtlich der Inflation, Diskussionspapier 8/96, Volkswirtschaftliche Forschungsgruppe der Deutschen Bundesbank.

Tanggaard, C. (1995). Nonparametric smoothing of yield curves, Working Paper D 95-1, Aarhus School of Business.

Waggoner, D. (1997). Spline methods for extracting interest rate curves from coupon bond prices, Working Paper 97-10, Federal Reserve Bank of Atlanta. 